\title{
Hipoelipticidade Gevrey de uma classe de sistemas sobredeterminados
}

\author{
Jairo Eloy Castellanos Ramos
}

TESE APRESENTADA

$\mathrm{AO}$

INSTITUTO DE MATEMÁTICA E ESTATÍSTICA

DA

UNIVERSIDADE DE SÃO PAULO

PARA

OBTENÇÃO DO GRAU DE DOUTOR

EM

MATEMÁTICA

Área de Concentração: Matemática

Orientador: Prof. Dr. Paulo Domingos Cordaro

Durante a elaboração deste trabalho o autor recebeu apoio financeiro da CAPES -São Paulo, 5 de outubro de 2001- 


\title{
Hipoelipticidade Gevrey de uma classe de sistemas sobredeterminados
}

\author{
Este exemplar corresponde à redação \\ final da tese devidamente corrigida e defendida \\ por Jairo Eloy Castellanos Ramos \\ e aprovada pela comissão julgadora.
}

-São Paulo, 5 de outubro de 2001-

Banca examinadora:

- Prof. Dr. Paulo Domingos Cordaro (orientador) (-IME-USP)

- Prof. Dr. Oscar Fortunato Vilcachagua Erazo (-IME-USP)

- Prof. Dr. Gerson Petronilho (-UFSCar)

- Prof. Dr. Adalberto Panobianco Bergamasco (-UFSCar)

- Prof. Dr. José Ruidival dos Santos Filho (-UFSCar) 
Ao meu filho Santiago.

Aos meus pais Julio e Altagracia.

À minha irmã Claudia (In memorian) $e$

À Sandra, companheira de todas as jornadas. 


\section{Agradecimentos}

Primeiramente, eu gostaria de agradecer ao Prof. Dr. Paulo Domingos Cordaro pela sugestão inicial e pelas sugestões subseqüentes, ao longo do processo que deu origem a este trabalho. Agradeço pela orientação e paciência durante estes cinco anos. Impossível descrever aqui o quanto aprendi enquanto estive sob sua orientação.

\section{Agradeço}

Ao prof. Dr. Oscar Vilcachagua Erazo pela boa vontade que sempre teve para discutir assuntos relacionados a este trabalho.

Aos meus colegas de Mestrado e Doutorado que muito me ajudaram e estimularam, com palavras e ações. Em especial, àqueles que comigo participaram do seminário sobre equações diferenciais parciais lineares, o qual se revelou muito útil na elaboração deste trabalho.

Aos meus amigos do IME, em especial Regina e Héliton, Claudia e Edwin, Alice e Leandro, Deborah, Laura, Sebastião, Júlio, Mário, Celso. 
Aos meus amigos colombianos, Liliam, Luz Estela, Patricia, Ivonne, Gladys, Paola, Alvaro, Hernan, Leonardo e Juan Diego. Em especial, agradeço a Sandra, ao Domingo e Mauricio pelo apoio e força, que me ajudaram a nunca desistir.

Um parágrafo especial quero dedicar aos meus professores que tive na Colômbia, com quem aprendi muito, e que para mim foram exemplo de dedicação ao trabalho. Por terem feito com que eu gostasse cada vez mais de matemática, e sobretudo com que eu aprendesse cada vez mais, agradeço ao professores Jorge Mejía, Pedro Isaza, Volker Stallbohm e Gilberto Garcia.

À CAPES pelo auxílio financeiro.

Finalizando, agradeço ao amor da Sandra, sua compreensão e apoio que tornaram mais fácil esta tarefa. 


\section{Resumo}

Neste trabalho apresentamos o estudo da hipoelipticidade Gevrey de uma classe de sistemas sobredeterminados de campos vetoriais utilizando as idéias de H. M. Maire. O sistema em questão é dado por $\mathbb{D}=d_{t}+d_{t} B\left(t, D_{x}\right)$, onde $B(t, \xi) \in \mathcal{C}^{\infty}\left(\Theta, \mathbf{R}^{m}\right)$.

\section{Abstract}

In this work we present the study of the hypoellipticity Gevrey of a class of overdetermined system of vector fields using the ideas of H. M. Maire. The system we are dealing with is $\mathbb{D}=d_{t}+d_{t} B\left(t, D_{x}\right)$, where $B(t, \xi) \in \mathcal{C}^{\infty}\left(\Theta, \mathbf{R}^{m}\right)$. 


\section{Sumário}

$\begin{array}{ll}\text { Introdução } & 1\end{array}$

1 Hipoelipticidade $\mathbb{G}^{s}$-Global 3

1.1 Notações e Definições . . . . . . . . . . . . . . . . . . . . . 3

1.2 O conceito de hipoelipticidade $\mathbb{G}^{s} \ldots \ldots \ldots \ldots$

1.3 Uma caracterização da hipoelipticidade $\mathbb{G}^{s} \ldots \ldots$. . . . . . 6

1.4 Uma condição necessária e suficiente para hipoelipticidade $\mathbb{G}^{s} \quad 14$

2 Hipoelipticidade Gevrey quando a Estrutura é Tubular 28

2.1 Classes de Gevrey . . . . . . . . . . . . . . . . . . . . . . . . . 28

2.2 Estruturas localmente integráveis e seu complexo. Estruturas tubulares e estruturas hipocomplexas . . . . . . . . . . 30

2.3 As classes $\mathrm{G}_{\mathrm{ML}}^{\mathrm{s}} \ldots \ldots \ldots \ldots$. . . . . . . . . . . . . 33

2.4 O conceito de hipoelipticidade Gevrey . . . . . . . . . . . . 34

2.5 O teorema principal . . . . . . . . . . . . . . 35

2.5.1 Demonstração do teorema principal:

Preliminares . . . . . . . . . . . . . . 36

2.5.2 Demonstração do teorema principal:

Conclusão. . . . . . . . . . . . . . . . . . . 39

3 Exemplo de um sistema que é analítico hipoelíptico, não hipoelíptico $\mathcal{C}^{\infty}$ e não $G^{s}$ hipoelíptico para $s>1$ 


\section{Introdução}

Neste trabalho apresentamos um estudo sobre a regularidade Gevrey das soluções do operador diferencial

$$
\mathbb{D}=d_{t}+d_{t} B\left(t, D_{x}\right),
$$

onde $t \in \Theta \subset \mathbf{R}^{n}, x \in \mathbf{R}^{m}$ e $B(t, \xi) \in \mathcal{C}^{\infty}\left(\Theta, \mathbf{R}^{m}\right)$. A pesquisa feita apoiou-se fortemente no trabalho [M] da bibliografia.

No capítulo I apresentamos uma condição necessária e suficiente para que $\mathbb{D}$ seja $\mathbb{G}^{s}$-globalmente hipoelíptico; dizemos que $\mathbb{D}$ é $\mathbb{G}^{s}$-globalmente hipoelíptico se $u \in \mathcal{C}^{\infty}\left(\Theta, \mathbf{L}^{2}(\Gamma(P))\right)$ e $\widehat{\mathbb{D} u} \in \Lambda^{1} \mathcal{W}^{s}(\Theta, P)$ implica $u \in$ $\mathcal{W}^{s}(\Theta, P)$. Mostramos que uma condição necessária e suficiente para que $\mathbb{D}$ seja $\mathbb{G}^{s}$-globalmente hipoelíptico é que a função $B(t, \xi) \in \mathcal{C}^{\infty}\left(\Theta, S^{m-1}\right)$ satisfaça a condição $(R)$ (Definição 1.6).

No capítulo II, introduzimos os espaços de funções Gevrey $G_{\mathrm{mL}}^{s, h}$. Isto é, dada uma carta $(\Omega ; Z)$ centrada na origem onde $Z=\left(Z_{1}, \ldots, Z_{m}\right)$ é uma aplicação infinitamente diferenciável, a valores em $\mathbb{C}^{m}$, com diferenciais $\mathrm{d} Z_{1}, \ldots, \mathrm{d} Z_{m}$

$\mathbb{C}$-linearmente independentes sobre $\Omega$. Coordenadas especiais $(x, t)=\left(x_{1}, \ldots\right.$, 
$\left.x_{m}, t_{1}, \ldots, t_{n}\right)$ em $\Omega$ permitem expressar $Z(x, t)$ na forma $x+i \Phi(x, t)$ tornando $\Omega$ difeomorfo ao traço $\Sigma \subset \mathbb{C}^{m} \times \mathbb{R}^{n}$ da aplicação $(x, t) \stackrel{\lambda}{\mapsto}(Z(x, t), t)$. Damos especial destaque para a base $(\mathrm{M}, \mathrm{L})$ de $\mathbb{C T} \Omega$ dual da base $(\mathrm{d} Z, \mathrm{~d} t)=$ $\left(\mathrm{d} Z_{1}, \ldots, \mathrm{d} Z_{m}, \mathrm{~d} t_{1}, \ldots, \mathrm{d} t_{n}\right)$ de $\mathbb{C} T^{*} \Omega$, constituída por derivações $\mathrm{M}_{1}, \ldots, \mathrm{M}_{m}$, $\mathrm{L}_{1}, \ldots, \mathrm{L}_{n}$ satisfazendo a dualidade $\mathrm{M}_{k} t_{j}=0, \mathrm{~L}_{j} Z_{k}=0, \mathrm{M}_{k} Z_{k^{\prime}}=\delta_{k k^{\prime}}$, $L_{j} t_{j^{\prime}}=\delta_{j j^{\prime}}$; aqui $\delta$ é a função delta de Kronecker. Funções $u=u(x, t)$ infinitamente diferenciáveis no fecho de vizinhanças $U \subset \subset \Omega$ da origem, cumprindo a estimativa

$$
\sup _{\bar{U}}\left|\mathrm{M}^{\alpha} \mathrm{L}^{\gamma} u\right| \leq \mathrm{const} \cdot h^{|\alpha|+|\gamma|} \cdot(|\alpha|+|\gamma|) !^{s}, \forall(\alpha, \gamma) \in \mathrm{Z}_{+}^{m+n}
$$

para uma amplitude $h>0$, constituem espaços de Banach $\mathrm{G}_{\mathrm{ML}}^{s, h}(U)$ com a respectiva norma $\|u\|_{s, h, U}$ determinada pelo ínfimo das constantes na estimativa. Como no caso usual, as inclusões $\mathrm{G}_{\mathrm{ML}}^{s, h}(U) \hookrightarrow \mathrm{G}_{\mathrm{ML}}^{s, h_{+}}(U)$ são compactas para todo $h<h_{+}$, tornando $\mathrm{G}_{\mathrm{ML}}^{s}(U)=\lim _{h \rightarrow \infty} \mathrm{G}_{\mathrm{ML}}^{s, h}(U)$ Hausdorff e Montel com a topologia de limite indutivo.

O principal resultado deste capítulo afirma que se $\mathrm{L}=d_{t}-i d \Phi \wedge \frac{\partial}{\partial x}$ e $\Phi$ uma função $\mathrm{C}^{\infty}$ aberta satisfazendo a estimativa (2.8) então $L$ é $\mathrm{G}_{\mathrm{ML}}^{s}-$ hipoelíptico para todo $s \geq 1$ (cf. Teorema 2). Segue que se $\mathcal{V}$ é uma estrutura hipocomplexa tubular sobre $\Omega$ com $m=1$ então $\mathcal{V}$ é $G^{s}$-hipoelíptico para todo $s \geq 1$ (cf. Corolário 2.1), onde $G^{s}$ é o espaço de funções Gevrey usual.

Finalmente, no capítulo III, damos um exemplo de um sistema $\mathrm{L}=$ $\left\{\mathrm{L}_{1}, \mathrm{~L}_{2}\right\}$ para o qual não se cumpre a propriedade:

$$
u \in C^{0}, \mathrm{~L} u \in \wedge^{1} G^{s} \Rightarrow u \in C^{1}, \quad s>1 .
$$




\section{Capítulo 1}

\section{Hipoelipticidade $\mathbb{G}^{s}$-Global}

\subsection{Notações e Definições}

Iniciamos esta seção introduzindo alguns espaços de funções.

Definição 1.1 Dados os números reais $s \geq 1, c>0$ e o conjunto compacto $P \subset S^{m-1}$, define-se o espaço de Hilbert $\mathbf{H}^{s, c}(P)$ como sendo o espaço das funções u pertencentes $a \mathbf{L}^{2}\left(\Gamma(P), e^{2 c|\xi|^{1 / s}} d \xi\right)$ com $\Gamma(P)$ o cone de base $P$ em $\mathbf{R}^{m}$.

Observação 1 As inclusões

$$
\mathbf{H}^{s, c}(P) \hookrightarrow \mathbf{H}^{s, c^{\prime}}(P) \quad, \quad c^{\prime}<c
$$

são contínuas com norma $\leq 1$ e portanto são fracamente compactas.

Segue da Observação 1 e de ([Ko], Th. 6 (pág. 372)) que os espaços

$$
\mathbf{H}^{s}(P)=\underset{c \searrow 0}{\lim _{c 0}} \mathbf{H}^{s, c}(P)
$$

são Hausdorff, reflexivos, completos, (DF) e bornológicos com a topologia de limite indutivo. 
Definição 1.2 Sejam $\Theta \subset \mathbf{R}^{n}$ aberto, $P \subset S^{m-1}$ compacto, $s \geq 1$ e $c>0$. Chamamos de $\mathcal{W}^{s, c}(\Theta, P)$ o conjunto das funções infinitamente diferenciáveis em $\Theta$ a valores no espaço de Hilbert $\mathbf{H}^{s, c}(P)$.

A topologia em $\mathcal{W}^{s, c}(\Theta, P)$ é dada pelas semi-normas

$$
\mathcal{P}_{l, K}^{s, c}(u)=\sum_{|\alpha| \leq l} \sup _{t \in K}\left[\int_{\Gamma(P)} e^{2 c|\xi|^{1 / s}}\left|\partial_{t}^{\alpha} u(t, \xi)\right|^{2} d \xi\right]^{1 / 2}, \quad K \subset \subset \Theta, l \in \mathbf{Z}_{+},
$$

que torna $\mathcal{W}^{s, c}(\Theta, P)$ um espaço de Fréchet.

Definição 1.3 Sejam $\Theta, P$ e s como na Definição 1.2. Vamos denotar por $\mathcal{W}^{s}(\Theta, P)$ o espaço das funções que pertencem a $\mathcal{W}^{s, c}(\Theta, P)$ para algum $c>0$.

Explicitando

$$
\mathcal{W}^{s}(\Theta, P) \doteq \bigcup_{c>0} \mathcal{W}^{s, c}(\Theta, P) \subset \mathcal{C}^{\infty}\left(\Theta, H^{s}(P)\right)
$$

e vamos considerar em $\mathcal{W}^{s}(\Theta, P)$ a topologia induzida por $\mathcal{C}^{\infty}\left(\Theta, H^{s}(P)\right)$.

\section{$1.2 \mathrm{O}$ conceito de hipoelipticidade $\mathbb{G}^{s}$}

Iniciamos introduzindo o operador $\mathbb{D}$ amplamente estudado por $\mathbf{F}$. Treves em [T]. Sejam $\Theta \subset \mathbf{R}^{n}$ aberto e $\phi \in \mathcal{C}^{\infty}\left(\Theta, \mathbf{R}^{m}\right)$. Vamos considerar o operador

$$
\mathbb{D}: \mathcal{D}^{\prime}\left(\Theta \times \mathbf{R}^{m}\right) \longrightarrow \wedge^{1} \mathcal{D}^{\prime}\left(\Theta \times \mathbf{R}^{m}\right),
$$

onde $\mathbb{D} u=d_{t} u+d_{t} B\left(t, D_{x}\right) u, t \in \Theta, x \in \mathbf{R}^{m}$ e $B(t, \xi)=\phi(t) \cdot \xi$. Observe que a equação

$$
\mathbb{D} u=f=\sum_{j=1}^{n} f_{j}(t, x) d t_{j}
$$


é equivalente ao sistema de primeira ordem

$$
\frac{\partial u}{\partial t_{j}}+\frac{\partial B}{\partial t_{j}}\left(t, D_{x}\right) u=f_{j} \quad j=1,2, \cdots, n .
$$

Explicitando,

$$
\begin{aligned}
\frac{\partial u}{\partial t_{j}}+\frac{\partial B}{\partial t_{j}}\left(t, D_{x}\right) u & =\frac{\partial u}{\partial t_{j}}+\left(\frac{\partial \phi}{\partial t_{j}} \cdot D_{x}\right) u \\
& =\frac{\partial u}{\partial t_{j}}+\frac{1}{i} \sum_{k=1}^{m} \frac{\partial \phi_{k}}{\partial t_{j}} \frac{\partial u}{\partial x_{k}} \\
& =f_{j} \quad j=1,2, \cdots, n
\end{aligned}
$$

Definição 1.4 Dizemos que o operador $\mathbb{D}$ é $\mathbb{G}^{s}$-Globalmente Hipoelíptico no indice $s$ em $\Theta \times P$ se para $u \in \mathcal{C}^{\infty}\left(\Theta, \mathbf{L}^{2}(\Gamma(P))\right)$,

$$
\widehat{\mathbb{D}} u \in \wedge^{1} \mathcal{W}^{s}(\Theta, P) \Longrightarrow u \in \mathcal{W}^{s}(\Theta, P),
$$

onde $\widehat{\mathbb{D}}=e^{-B(t, \xi)} d_{t} e^{B(t, \xi)}$.

Observação 2 Se $\mathcal{O} \subset \Theta$ aberto e $u \in \mathcal{D}^{\prime}\left(\mathcal{O} ; \mathbf{L}^{2}(\Gamma(P))\right)$, então $\mathbb{D} u \in$ $\wedge^{1} \mathcal{C}^{\infty}\left(\mathcal{O} ; \mathbf{L}^{2}(\Gamma(P))\right) \Longrightarrow u \in \mathcal{C}^{\infty}\left(\mathcal{O} ; \mathbf{L}^{2}(\Gamma(P))\right)$. A prova consiste em aproveitar-se das equações (1.2), agora assumidas válidas em $\mathcal{O}$, onde $f \in$ $\mathcal{C}^{\infty}\left(\mathcal{O} ; \mathbf{L}^{2}(\Gamma(P))\right)$, para obter regularidade arbitrariamente alta com respeito a $t$ da solução $u$.

Definição 1.50 operador $\mathbb{D}$ é $\mathbb{G}^{s}$ - Hipoelíptico em $\Theta \times P$ se $\mathbb{D}$ é $\mathbb{G}^{s}-$ globalmente hipoelíptico em cada subconjunto $\mathcal{O} \times P$ de $\Theta \times P$ com $\mathcal{O} \subset \Theta$ aberto. 


\subsection{Uma caracterização da hipoelipticidade $\mathbb{G}^{s}$}

Vamos agora a estudar uma caracterização da hipoelipticidade $\mathbb{G}$ do operador $\mathbb{D}$, na sua demonstração usaremos os seguintes Lemas:

Lema 1 Seja $s \geq 1$. Dada $\psi \in \mathcal{C}^{\infty}\left(\Theta, \mathrm{L}^{2}(\Gamma(P))\right)$ com $\widehat{\mathbb{D}} \psi \in \wedge^{1} \mathcal{W}^{s}(\Theta, P)$, existem $\psi_{j} \in \mathcal{C}^{\infty}\left(\Theta ; \mathbf{L}_{c}^{2}(\Gamma(P))\right)$ tais que $\psi_{j} \rightarrow \psi$ em $\mathcal{C}^{\infty}\left(\Theta, \mathbf{L}^{2}(\Gamma(P))\right)$ e $\widehat{\mathbb{D}} \psi_{j} \rightarrow \widehat{\mathbb{D}} \psi$ em $\wedge^{1} \mathcal{W}^{s}(\Theta, P)$.

\section{Demonstração :}

$\triangleright \quad$ Seja $\mathcal{X}_{j}$ a função característica do conjunto $\Gamma(P) \cap\{|\xi| \leq j\}$ e considerando as funções $\psi_{j}=\psi(t, \xi) \mathcal{X}_{j}(\xi), t \in \Theta, \xi \in \mathbf{R}^{m}, j=1,2, \ldots$ Vê-se facilmente que $\psi_{j} \in \mathcal{C}^{\infty}\left(\Theta ; \mathbf{L}_{c}^{2}\left(\mathbf{R}^{m}\right)\right)$ e $\quad \psi_{j} \stackrel{j \rightarrow \infty}{\longrightarrow} \psi$ em $\mathcal{C}^{\infty}\left(\Theta, \mathbf{L}^{2}(\Gamma(P))\right)$.

Por outro lado, observe que $\widehat{\mathbb{D}} \psi \in \wedge^{1} \mathcal{W}^{s, c}(\Theta, P)$ para algum $c>0$. Então, para $c^{\prime}<c, c^{\prime}>0, K \subset \Theta$ compacto e $l \geq 0$, temos que

$$
\begin{aligned}
& \mathcal{P}_{l, K}^{s, c^{\prime}}\left(\widehat{\mathbb{D}} \psi-\widehat{\mathbb{D}} \psi_{j}\right)=\sum_{|\alpha| \leq l} \sup _{t \in K}\left[\int_{\Gamma(P)} e^{2 c^{\prime}|\xi|^{1 / s}}\left|\partial_{t}^{\alpha}\left[\widehat{\mathbb{D}} \psi-\widehat{\mathbb{D}} \psi_{j}\right](t, \xi)\right|^{2} d \xi\right]^{1 / 2} \\
& =\sum_{|\alpha| \leq l} \sup _{t \in K}\left[\int_{\Gamma(P)} e^{2 c^{\prime}|\xi|^{1 / s}}\left|\partial_{t}^{\alpha} \widehat{\mathbb{D}} \psi(t, \xi)\right|^{2}\left|1-\mathcal{X}_{j}(\xi)\right|^{2} d \xi\right]^{1 / 2} \\
& =\sum_{|\alpha| \leq l} \sup _{t \in K}\left[\int_{\Gamma(P)} e^{2\left(c^{\prime}-c\right)|\xi|^{1 / s}} e^{2 c|\xi|^{1 / s}}\left|\partial_{t}^{\alpha} \widehat{\mathbb{D}} \psi(t, \xi)\right|^{2}\left|1-\mathcal{X}_{j}(\xi)\right|^{2} d \xi\right]^{1 / 2} \\
& \leq \sum_{|\alpha| \leq l} \sup _{t \in K}\left[\int_{\Gamma(P)} e^{2\left(c^{\prime}-c\right)|j|^{1 / s}} e^{2 c|\xi|^{1 / s}}\left|\partial_{t}^{\alpha} \widehat{\mathbb{D} \psi}(t, \xi)\right|^{2}\left|1-\mathcal{X}_{j}(\xi)\right|^{2} d \xi\right]^{1 / 2} \\
& \leq e^{\left(c^{\prime}-c\right)|j|^{1 / s}} \sum_{|\alpha| \leq l} \sup _{t \in K}\left[\int_{\Gamma(P)} e^{2 c|\xi|^{1 / s}}\left|\partial_{t}^{\alpha} \widehat{\mathbb{D} \psi}(t, \xi)\right|^{2} d \xi\right]^{1 / 2},
\end{aligned}
$$

que tende a zero quando $j \rightarrow \infty$, pois $\widehat{\mathbb{D}} \psi \in \wedge^{1} \mathcal{W}^{s, c}(\Theta, P)$. 
Lema 2 Seja $G$ um espaço localmente convexo, Hausdorff e seja $\left\{E_{n}\right\}$ uma seqüência de espaços de Fréchet. Seja F um espaço de Fréchet e assuma dadas aplicações lineares contínuas $u_{n}: E_{n} \longrightarrow G, u: F \longrightarrow G$ tais que

$$
u(F) \subset \bigcup_{n} u_{n}\left(E_{n}\right)
$$

Então existe $k$ tal que $u(F) \subset u_{k}\left(E_{k}\right)$.

\section{Demonstração :}

$\triangleright \quad$ Ver [G]. Teorema 1, pág. 198.

Notação: Para $L \subset \Theta$ compacto e $l$ inteiro não negativo denotamos por

$$
\mathcal{Q}_{l, L}(f)=\sum_{|\alpha| \leq l} \sup _{t \in L}\left\|\partial_{t}^{\alpha} f(t, \cdot)\right\|_{\mathbf{L}^{2}(\Gamma(P))} .
$$

Proposição 1.1 operador $\mathbb{D}$ é $\mathbb{G}^{s}$-globalmente hipoelíptico em $\Theta \times P$ se e só se para cada $c>0$, existe $c^{\prime}>0$ tal que para todo inteiro não negativo $m e$ todo compacto $K \subset \Theta$ existem $m^{\prime} \in \mathrm{N}, K^{\prime} \subset \Theta$ compacto e $C>0$ constante com

$$
\mathcal{P}_{m, K^{\prime}}^{s, c^{\prime}}(g) \leq C\left(\mathcal{Q}_{m^{\prime}, K^{\prime}}(g)+\mathcal{P}_{m^{\prime}, K^{\prime}}^{s, c}(\widehat{\mathbb{D}} g)\right), \quad \forall g \in \mathcal{C}^{\infty}\left(\Theta, \mathbf{L}_{c}^{2}(\Gamma(P))\right.
$$

\section{Demonstração :}

$\triangleright \quad[\Longrightarrow]$. Seja $F \doteq\left\{w \in \mathcal{C}^{\infty}\left(\Theta, \mathbf{L}^{2}(\Gamma(P))\right): \widehat{\mathbb{D}} w \in \wedge^{1} \mathcal{W}^{s, c}(\Theta, P)\right\}$.

Afirmação: Existe $c^{\prime}>0$ tal que $F \subset \mathcal{W}^{s, c^{\prime}}(\Theta, P)$. A afirmação se segue do Lema 2. De fato, $F$ é um espaço de Fréchet com a topologia do gráfico e as aplicações inclusões $\Pi$ de $F$ em $\mathcal{C}^{\infty}\left(\Theta, \mathbf{L}^{2}(\Gamma(P))\right)$ e $J_{n}: \mathcal{W}^{s, 1 / n}(\Theta, P) \hookrightarrow \mathcal{C}^{\infty}\left(\Theta, \mathrm{L}^{2}(\Gamma(P))\right)$ são contínuas, e por hipótese

$$
\Pi(F) \subset \bigcup J_{n}\left(\mathcal{W}^{s, 1 / n}(\Theta, P)\right)
$$

Assim, pelo Lema 2, existe $c^{\prime}>0$ tal que $F \subset \mathcal{W}^{s, c^{\prime}}(\Theta, P)$. 
Por outro lado, pelo teorema do gráfico fechado $F \hookrightarrow \mathcal{W}^{s, c^{\prime}}(\Theta, P)$ é contínua. Portanto se cumpre a condição (1.3).

$[\Longleftarrow]$. Seja $w \in \mathcal{C}^{\infty}\left(\Theta, \mathbf{L}^{2}(\Gamma(P))\right)$ tal que $\widehat{\mathbb{D}} w \in \wedge^{1} \mathcal{W}^{s}(\Theta, P)$. Logo existe $c>0$ tal que $\widehat{\mathbb{D}} w \in \wedge^{1} \mathcal{W}^{s, c}(\Theta, P)$. Pelo Lema 1 existem $w_{j} \in$ $\mathcal{C}^{\infty}\left(\Theta, \mathbf{L}_{c}^{2}(\Gamma(P))\right)$ tais que $w_{j} \rightarrow w$ em $\mathcal{C}^{\infty}\left(\Theta, \mathbf{L}^{2}(\Gamma(P))\right)$ e $\widehat{\mathbb{D}} w_{j} \rightarrow \widehat{\mathbb{D}} w$ em $\wedge^{1} \mathcal{W}^{s, c}(\Theta, P)$. Aplicando a desigualdade (1.3) para a função $w_{j}-w_{k}$ obtemos que existe $c^{\prime}>0$ tal que dados $m$ inteiro não negativo e $K \subset \Theta$ compacto existem $m^{\prime} \in \mathbb{N}, K^{\prime} \subset \Theta$ compacto e $C>0$ constante com

$$
\mathcal{P}_{m, K}^{s, c^{\prime}}\left(w_{j}-w_{k}\right) \leq C\left(\mathcal{Q}_{m^{\prime}, K^{\prime}}\left(w_{j}-w_{k}\right)+\mathcal{P}_{m^{\prime}, K^{\prime}}^{s, c}\left(\widehat{\mathbb{D}}\left[w_{j}-w_{k}\right]\right)\right)
$$

Como $\left\{w_{j}\right\}$ e $\left\{\widehat{\mathbb{D}} w_{j}\right\}$ são Cauchy em $\mathcal{C}^{\infty}\left(\Theta ; \mathbf{L}_{c}^{2}(\Gamma(P))\right)$ e $\wedge^{1} \mathcal{W}^{s, c}(\Theta, P)$ respectivamente, $\forall(m, K)$

$$
\mathcal{P}_{m, K}^{s, c^{\prime}}\left(w_{j}-w_{k}\right) \underset{j, k \rightarrow \infty}{\longrightarrow} 0
$$

e $\operatorname{assim}\left\{w_{j}\right\}$ é Cauchy em $\mathcal{W}^{s, c^{\prime}}(\Theta, P)$.

Portanto $w_{j} \rightarrow w^{\bullet}$ em $\mathcal{W}^{s, c^{\prime}}(\Theta, P) \hookrightarrow \mathcal{C}^{\infty}\left(\Theta ; \mathbf{L}^{2}(\Gamma(P))\right)$ e assim $w=$ $w^{\circ}$.

Proposição 1.20 operador $\mathbb{D}$ é $\mathbb{G}^{s}$-globalmente hipoelíptico em $\Theta \times P$ se e só se para cada $c>0$ existe $c^{\prime}>0$ tal que para todo $K \subset \Theta$ compacto existem $K^{\prime} \subset \Theta$ compacto, $m^{\prime}>0$ inteiro e $C>0$ constante tais que

$$
\begin{aligned}
e^{c^{\prime} \rho^{1 / s}} \sup _{t \in K} e^{-\rho B(t, \dot{\xi})}|v(t)| \leq C\left\{\sup _{t \in K^{\prime}} e^{-\rho B(t, \dot{\xi})}|v(t)|\right. & \\
& \left.+\sum_{|\alpha| \leq m^{\prime}} e^{c \rho^{1 / s}} \sup _{t \in K^{\prime}} e^{-\rho B(t, \dot{\xi})}\left|\partial_{t}^{\alpha} d v(t)\right|\right\}
\end{aligned}
$$

$\forall \rho>0, \quad \forall \dot{\xi} \in P, \quad \forall v \in \mathcal{C}^{\infty}(\Theta)$. 


\section{Demonstração :}

Começaremos demonstrando que a propriedade (1.4) é equivalente a:

Para cada $c>0$ existe $c^{\prime}>0$ tais que para todo $K \subset \Theta$ compacto $e$ $m \in \mathbb{N}$ existem $K^{\prime} \subset \Theta$ compacto, $m^{*}>0$ inteiro $e C^{*}>0$ constante tal que

$$
\begin{aligned}
& \sum_{|\alpha| \leq m} e^{c^{\prime} \rho^{1 / s}} \sup _{t \in K} e^{-\rho B(t, \dot{\xi})}\left|\partial_{t}^{\alpha} v(t)\right| \leq C^{*}\left\{\sup _{t \in K^{\prime}} e^{-\rho B(t, \dot{\xi})}|v(t)|\right. \\
& \left.\quad+\sum_{|\alpha| \leq m^{*}} e^{c \rho^{1 / s}} \sup _{t \in K^{\prime}} e^{-\rho B(t, \dot{\xi})}\left|\partial_{t}^{\alpha} d v(t)\right|\right\}, \rho>0, \dot{\xi} \in P, v \in \mathcal{C}^{\infty}(\Theta) .
\end{aligned}
$$

É imediato que (1.5) implica (1.4), basta tomar $m=0$.

Vejamos que (1.4) implica (1.5). Basta provar que para $|\gamma| \leq m$

$$
\begin{aligned}
& e^{c^{\prime} \rho^{1 / s}} \sup _{t \in K} e^{-\rho B(t, \dot{\xi})}\left|\partial_{t}^{\gamma} v(t)\right| \leq C^{*}\left\{\sup _{t \in K^{\prime}} e^{-\rho B(t, \dot{\xi})}|v(t)|\right. \\
& \left.+\sum_{|\alpha| \leq m^{*}} e^{c \rho^{1 / s}} \sup _{t \in K^{\prime}} e^{-\rho B(t, \dot{\xi})}\left|\partial_{t}^{\alpha} d v(t)\right|\right\}
\end{aligned}
$$

Aplicando (1.4) $\operatorname{com} \partial_{t}^{\gamma} v$ em lugar de $v$ obtemos que para $c>0$ existe $c^{\prime}>0$ tal que para cada $K \subset \Theta$ compacto existem $K^{\prime} \subset \Theta$ compacto, $m^{\prime}>0$ inteiro e $C>0$ constante tais que

$$
\begin{aligned}
e^{c^{\prime} \rho^{1 / s}} \sup _{t \in K} e^{-\rho B(t, \dot{\xi})}\left|\partial_{t}^{\gamma} v(t)\right| \leq & C\left\{\sup _{t \in K^{\prime}} e^{-\rho B(t, \dot{\xi})}\left|\partial_{t}^{\gamma} v(t)\right|\right. \\
& \left.+\sum_{|\alpha| \leq m^{\prime}} e^{c \rho^{1 / s}} \sup _{t \in K^{\prime}} e^{-\rho B(t, \dot{\xi})}\left|\partial_{t}^{\gamma+\alpha} d v(t)\right|\right\}
\end{aligned}
$$

e portanto, existem $C^{*}$ e $m^{*}=m^{\prime}+m$ tal que

$$
e^{c^{\prime} \rho^{1 / s}} \sup _{t \in K} e^{-\rho B(t, \dot{\xi})}\left|\partial_{t}^{\gamma} v(t)\right| \leq C^{*} \sum_{|\alpha| \leq m^{*}} e^{c \rho^{1 / s}} \sup _{t \in K^{\prime}} e^{-\rho B(t, \dot{\xi})}\left|\partial_{t}^{\alpha} d v(t)\right|
$$


Podemos agora demonstrar a proposição: Basta provar que (1.5) é equivalente a (1.3).

Vejamos que (1.5) implica (1.3). Escolha $v$ dependendo de $\xi=\rho \dot{\xi}$ :

$$
v(t, \xi)=e^{B(t, \xi)} u(t, \xi), u \in \mathcal{C}^{\infty}\left(\Theta, \mathbf{L}_{c}^{2}(\Gamma(P))\right)
$$

Seja $c>0$ e escolha $c^{*}<c$. Com estas escolhas de $v$ e $c^{*},(1.5)$ implica que existe $c^{* *}$ tal que dados $K \subset \Theta$ compacto e $m \in \mathrm{N}$ existem $K^{\prime} \subset \Theta$ compacto, $m^{*}>0$ inteiro e $C^{*}>0$ constante tais que

$$
\begin{aligned}
& \sum_{|\alpha| \leq m} e^{c^{* *}|\xi|^{1 / s}} \sup _{t \in K} e^{-\rho B(t, \dot{\xi})}\left|\partial_{t}^{\alpha} e^{\rho B(t, \dot{\xi})} u(t, \xi)\right| \\
& \leq C^{*}\left\{\sup _{t \in K^{\prime}}|u(t, \xi)|+\sum_{|\alpha| \leq m^{*}} e^{c^{*}|\xi|^{1 / s}} \sup _{t \in K^{\prime}} e^{-\rho B(t, \dot{\xi})}\left|\partial_{t}^{\alpha} d_{t} e^{B(t, \xi)} u(t, \xi)\right|\right\} \\
& =C^{*}\left\{\sup _{t \in K^{\prime}}|u(t, \xi)|+\sum_{|\alpha| \leq m^{*}} e^{c^{*}|\xi|^{1 / s}} \sup _{t \in K^{\prime}} e^{-\rho B(t, \dot{\xi})}\left|\partial_{t}^{\alpha} e^{\rho B(t, \dot{\xi})} \widehat{\mathbb{D}} u(t, \xi)\right|\right\} \\
& \leq C_{1}\left\{\sup _{t \in K^{\prime}}|u(t, \xi)|+\sum_{|\alpha| \leq m^{\prime \prime}} \rho^{m^{\prime \prime}} e^{c^{*}|\xi|^{1 / s}} \sup _{t \in K^{\prime}}\left|\partial_{t}^{\alpha} \widehat{\mathbb{D}} u(t, \xi)\right|\right\} \\
& \leq C_{1}\left\{\sup _{t \in K^{\prime}}|u(t, \xi)|+\sum_{|\alpha| \leq m^{\prime \prime}} e^{c|\xi|^{1 / s}} \sup _{t \in K^{\prime}}\left|\partial_{t}^{\alpha} \widehat{\mathbb{D}} u(t, \xi)\right|\right\}
\end{aligned}
$$

para certos $C_{1}>0$ e $m^{\prime \prime} \in \mathbf{Z}_{+}$onde $\widehat{\mathbb{D}}=e^{-B(t, \xi)} d_{t} e^{B(t, \xi)}$.

Por outro lado, observe que para $c^{\prime}<c^{* *}$ :

$$
\begin{aligned}
\sum_{|\alpha| \leq m} e^{c^{\prime}|\xi|^{1 / s}} \sup _{t \in K} & \left|\partial_{t}^{\alpha} u(t, \xi)\right| \\
& \leq \sum_{|\alpha| \leq m} e^{c^{* *}|\xi|^{1 / s}} \sup _{t \in K} e^{-\rho B(t, \dot{\xi})}\left|\partial_{t}^{\alpha} e^{\rho B(t, \dot{\xi})} u(t, \xi)\right|
\end{aligned}
$$


Portanto, usando (1.7), obtemos

$$
\begin{aligned}
\sum_{|\alpha| \leq m} e^{c^{\prime}|\xi|^{1 / s}} \sup _{t \in K}\left|\partial_{t}^{\alpha} u(t, \xi)\right| \leq & C_{1}\left\{\sup _{t \in K^{\prime}}|u(t, \xi)|\right. \\
& \left.+\sum_{|\alpha| \leq m^{\prime \prime}} e^{c|\xi|^{1 / s}} \sup _{t \in K^{\prime}}\left|\partial_{t}^{\alpha} \widehat{\mathbb{D}} u(t, \xi)\right|\right\} .
\end{aligned}
$$

A desigualdade (1.3) segue disto, depois de tomar o quadrado e integrar sobre $\Gamma(P)$.

Vejamos que (1.3) implica (1.5). Sejam $c>0$ e

$$
u(t, \xi)=e^{-B(t, \xi)} v(t) \phi(\xi), \quad v \in \mathcal{C}^{\infty}(\Theta)
$$

e $\phi \in \mathcal{C}_{0}^{\infty}\left(\mathbf{R}^{m}\right)$ com suporte em $\Gamma(P)$.

Aplicando a propriedade (1.3) para $c^{*}<c$ e $u$, temos que $\forall(K, m)$ existem $\left(K^{\prime}, m^{\prime}\right)$ tais que

$$
\begin{aligned}
\sum_{|\alpha| \leq m} \sup _{t \in K}\left[\int_{\Gamma(P)} e^{2 c^{* \prime}|\xi|^{1 / s}}\left|\partial_{t}^{\alpha}\left(e^{-B(t, \xi)} v(t)\right) \phi(\xi)\right|^{2} d \xi\right]^{1 / 2} \\
\leq C\left\{\sum_{|\alpha| \leq m^{\prime}} \sup _{t \in K^{\prime}}\left[\int_{\Gamma(P)}\left|\partial_{t}^{\alpha}\left(e^{-B(t, \xi)} v(t)\right)\right|^{2}|\phi(\xi)|^{2} d \xi\right]^{1 / 2}\right. \\
\left.+\sum_{|\alpha| \leq m^{\prime}} \sup _{t \in K^{\prime}}\left[\int_{\Gamma(P)} e^{2 c^{*}|\xi|^{1 / s}}\left|\partial_{t}^{\alpha} \widehat{\mathbb{D}} e^{-B(t, \xi)} v(t)\right|^{2}|\phi(\xi)|^{2} d \xi\right]^{1 / 2}\right\} .
\end{aligned}
$$

Para qualquer $\dot{\xi}_{0} \in P, \rho>0$ tomando, na desigualdade anterior, a seqüência $\phi_{j} \in \mathcal{C}^{\infty}\left(\mathbf{R}^{m}\right)$ com suporte contido em $\Gamma(P)$ tal que $\operatorname{supp} \phi_{j} \rightarrow$ $\left\{\rho \dot{\xi}_{0}\right\}$ e $\phi_{j}^{2} \rightarrow \delta\left(\xi-\rho \dot{\xi}_{0}\right)$ quando $j \rightarrow \infty$ em $\mathcal{E}^{\prime}\left(\mathbf{R}^{m}\right)$, obtemos 


$$
\begin{aligned}
\sum_{|\alpha| \leq m} \sup _{t \in K}\left[e^{2 c^{* \prime}|\rho|^{1 / s}}\left|\partial_{t}^{\alpha}\left(e^{-B\left(t, \rho \dot{\xi}_{0}\right)} v(t)\right)\right|^{2}\right]^{1 / 2} \\
\leq C\left\{\sum_{|\alpha| \leq m^{\prime}} \sup _{t \in K^{\prime}}\left[\left|\partial_{t}^{\alpha}\left(e^{-B\left(t, \rho \dot{\xi}_{0}\right)} v(t)\right)\right|^{2}\right]^{1 / 2}\right. \\
\left.\quad+\sum_{|\alpha| \leq m^{\prime}} \sup _{t \in K^{\prime}}\left[e^{2 c^{*}|\rho|^{1 / s}}\left|\partial_{t}^{\alpha}\left(e^{-B\left(t, \rho \dot{\xi}_{0}\right)} d v(t)\right)\right|^{2}\right]^{1 / 2}\right\} .
\end{aligned}
$$

Assim para $\rho>1$ temos

$$
\begin{aligned}
& \left.\sum_{|\alpha| \leq m} e^{c^{* \prime}|\rho|^{1 / s}} \sup _{t \in K} \mid \partial_{t}^{\alpha}\left(e^{-B(t, \rho \dot{\xi} 0}\right) v(t)\right) \mid \\
& \leq C\left\{\sup _{t \in K^{\prime}} \sum_{|\alpha| \leq m^{\prime}} \mid \partial_{t}^{\alpha}\left(e^{-B\left(t, \rho \dot{\xi}_{0}\right)} v(t)\right)\right) \mid \\
& \left.+\sum_{|\alpha| \leq m^{\prime}} e^{c^{*}|\rho|^{1 / s}} \sup _{t \in K^{\prime}}\left|\partial_{t}^{\alpha}\left(e^{-B\left(t, \rho \dot{\xi}_{0}\right)} d v(t)\right)\right|\right\} \\
& \leq C_{1}\left\{\rho^{m^{\prime}} \sup _{t \in K^{\prime}}\left|e^{-B\left(t, \rho \dot{\xi}_{0}\right)} v(t)\right|\right. \\
& \left.+\sum_{|\alpha| \leq m^{\prime}} e^{c^{*}|\rho|^{1 / s}} \sup _{t \in K^{\prime}}\left|\sum_{|\beta| \leq|\alpha|} \partial_{t}^{\alpha-\beta}\left(e^{-B\left(t, \rho \dot{\xi}_{0}\right)}\right) \partial_{t}^{\beta} d v(t)\right|\right\} \\
& \leq C_{1}\left\{\rho^{m^{\prime}} \sup _{t \in K^{\prime}}\left|e^{-B\left(t, \rho \dot{\xi}_{0}\right)} v(t)\right|\right. \\
& \left.+\sum_{|\alpha| \leq m^{\prime}} e^{c^{*}|\rho|^{1 / s}} \sup _{t \in K^{\prime}}\left|\sum_{|\beta| \leq|\alpha|} \rho^{\alpha-\beta} e^{-B\left(t, \rho \dot{\xi}_{0}\right)} \partial_{t}^{\beta} d v(t)\right|\right\} \\
& \leq C_{2}\left\{\rho^{m^{\prime}} \sup _{t \in K^{\prime}}\left|e^{-B\left(t, \rho \dot{\xi}_{0}\right)} v(t)\right|\right. \\
& \left.+\sum_{|\alpha| \leq m^{\prime}} \rho^{m^{\prime}} e^{c^{*}|\rho|^{1 / s}} \sup _{t \in K^{\prime}}\left|e^{-B\left(t, \rho \dot{\xi}_{0}\right)} \partial^{\alpha} d v(t)\right|\right\} \\
& \leq C_{2}\left\{\rho^{m^{\prime}} \sup _{t \in K^{\prime}}\left|e^{-B\left(t, \rho \dot{\xi}_{0}\right)} v(t)\right|\right. \\
& \left.+\sum_{|\alpha| \leq m^{\prime}} e^{c|\rho|^{1 / s}} \sup _{t \in K^{\prime}}\left|e^{-B\left(t, \rho \dot{\xi}_{0}\right)} \partial_{t}^{\alpha} d v(t)\right|\right\}
\end{aligned}
$$

Portanto, para $c^{\prime}<c^{* \prime}$, temos que: 


$$
\begin{aligned}
& \sum_{|\alpha| \leq m} e^{c^{\prime}|\rho|^{1 / s}} \sup _{t \in K}\left|\partial_{t}^{\alpha}\left(e^{-B\left(t, \rho \dot{\xi}_{0}\right)} v(t)\right)\right| \leq C_{2}\left\{\sup _{t \in K^{\prime}}\left|e^{-B\left(t, \rho \dot{\xi}_{0}\right)} v(t)\right|\right. \\
& \left.\quad+\sum_{|\alpha| \leq m^{\prime}} e^{c|\rho|^{1 / s}} \sup _{t \in K^{\prime}}\left|e^{-B\left(t, \rho \dot{\xi}_{0}\right)} \partial_{t}^{\alpha} d v(t)\right|\right\} .
\end{aligned}
$$

Quando $0<\rho \leq 1$ (1.5) segue trivialmente. Assim, segue (1.5) no ponto $\rho \dot{\xi}_{0}$ com uma constante válida para qualquer $\rho>0, \dot{\xi} \in P$. 


\subsection{Uma condição necessária e suficiente para hipoelipticidade $\mathbb{G}^{s}$}

Agora introduzimos as funções $F_{K}$ e $E_{K, \mu}$ necessárias para medir a variação de $B$, que servirão para dar uma condição necessária e suficiente sobre $B$ para a $\mathbb{G}^{s}$-hipoelipticidade de $\mathbb{D}=d_{t}+d_{t} B\left(t, D_{x}\right)$.

Sejam $\Theta \subset \mathbf{R}^{n}$ aberto, $K$ um conjunto compacto em $\Theta$ e $(t, \dot{\xi}) \in K \times$ $S^{m-1}$; para $r \in \mathbf{R}_{+} \cup\{0\}$, definimos os conjuntos

$$
\begin{aligned}
K(t, \dot{\xi} ; r) & =\{s \in K ; B(s, \dot{\xi}) \leq B(t, \dot{\xi})+r\} \\
K(t, \dot{\xi}) & =K(t, \dot{\xi} ; 0) .
\end{aligned}
$$

Estes são subconjuntos de sub níveis fechados de $s \mapsto B(s, \dot{\xi})$ em $K$ de altura $B(t, \dot{\xi})+r$. Denotamos por $K_{0}(t, \dot{\xi} ; r)$ a componente conexa de $t$ em $K(t, \dot{\xi} ; r)$. Fazemos

$$
\begin{aligned}
F_{K}(t, \dot{\xi} ; r) & =\sup _{s \in K_{0}(t, \dot{\xi} ; r)}(B(t, \dot{\xi})-B(s, \dot{\xi})) \\
F_{K}(t, \dot{\xi}) & =F_{K}(t, \dot{\xi} ; 0) .
\end{aligned}
$$

Observe que a função $r \mapsto F_{K}(t, \dot{\xi} ; r)$ é não decrescente e não negativa. Além disso, se $d_{t} B(t, \dot{\xi}) \neq 0$ e $t$ é um ponto interior de $K$, então $F_{K}(t, \dot{\xi})>0$.

Observação 3 A função $t \mapsto F_{K}(t, \dot{\xi})$, em geral, não é contínua, nem semicontínua inferiormente; este fato não permite inferir que $\inf _{t} F_{K}(t, \dot{\xi})>0$ mesmo que $F_{K}(t, \dot{\xi})>0$ em cada ponto de um conjunto compacto. 
Lema $3[M]$ Seja $\left(t_{0}, \dot{\xi}_{0}\right) \in \Theta \times S^{m-1}$ e considere:

1. $t \rightarrow B\left(t, \dot{\xi}_{0}\right)$ tem um minimo local em $t_{0}$;

2. Existe uma vizinhança compacta $K \subset \Theta$ de $t_{0}$ tal que $F_{K}\left(t_{0}, \dot{\xi}\right)=0$.

Então (1) implica (2) e o recíproco vale se $K\left(t_{0}, \dot{\xi}_{0}\right)$ tem só um número finito de componentes conexas.

\section{Demonstração :}

$\triangleright \quad$ É evidente que 1. implica 2.. Vamos provar a outra parte; para $t_{0} \in$ $\Theta, K$ como em 2., a hipótese adicional implica a existência de uma vizinhança $U$ de $t_{0}$ tal que $U \cap K\left(t_{0}, \dot{\xi}_{0}\right)=U \cap K_{0}\left(t_{0}, \dot{\xi}_{0}\right)$. Então $B\left(t, \dot{\xi}_{0}\right) \geq B\left(t_{0}, \dot{\xi}_{0}\right)$ para $t \in U$ porque $F_{K}\left(t_{0}, \dot{\xi}_{0}\right)=0$ implica $B\left(t, \dot{\xi}_{0}\right)=$ $B\left(t_{0}, \dot{\xi}_{0}\right)$ para $t \in K\left(t_{0}, \dot{\xi}_{0}\right)$ e $B\left(t, \dot{\xi}_{0}\right)>B\left(t_{0}, \dot{\xi}\right)$ em $K / K\left(t_{0}, \dot{\xi}_{0}\right)$.

Observação: Se $\mathbb{D}$ é $\mathbb{G}^{s}$-hipoelíptico em $\Theta \times P$, então para todo $\dot{\xi} \in P$ a aplicação $t \mapsto B(t, \dot{\xi})$ não pode ter um mínimo local em $\Theta$. De fato, a Proposição 1.2 mostra isto facilmente: Suponha que $\mathbb{D}$ é $\mathbb{G}^{s}$-hipoelíptico e $B\left(t_{0}, \dot{\xi}_{0}\right) \leq B\left(t, \dot{\xi}_{0}\right)$ para $t$ em uma vizinhança $\mathcal{O}$ de $t_{0}$. Aplicando a condição (1.4) com $v=1$, obtemos que para $c=1$ existe $c^{\prime}>0$ tal que para $K=\left\{t_{0}\right\}$ existe uma constante $C>0$ tal que

$$
e^{c^{\prime} \rho^{1 / s}} e^{-\rho B\left(t_{0}, \dot{\xi}_{0}\right)} \leq C \sup _{t \in \mathcal{O}} e^{-\rho B\left(t, \dot{\xi}_{0}\right)}=C e^{-\rho B\left(t_{0}, \dot{\xi}_{0}\right)}
$$

e assim

$$
e^{c^{\prime} \rho^{1 / s}} \leq C \quad \forall \rho>0
$$

o que é um absurdo. 
Lema 4 Seja $K_{0}$ um subconjunto compacto conexo de $\Theta, B(t)$ uma função Lipschitz continua e a valores reais em $\Theta, \beta$ o máximo de $B$ sobre $K_{0}$.

Existe uma constante $C=C\left(B, K_{0}\right)>0$ tal que, dado quaisquer numero $\epsilon$, $0<\epsilon<\inf \left(1, \operatorname{dist}\left(K_{0}, \partial \Theta\right)\right)$, e qualquer dois pontos $t, t^{\prime}$ de $K_{0}$, existe um caminho de integração $\gamma$, linear por partes, contido em $\Theta$, juntando $t$ a $t^{\prime} e$ tendo as seguintes propriedades:

1. $\gamma$ esta contido numa $\epsilon$-vizinhança de $K_{0}$;

2. para todo $s \in \gamma, B(s) \leq \beta+\epsilon$;

3. o comprimento de $\gamma$ não excede $C \epsilon^{1-n}$.

\section{Demonstração :}

$\triangleright \quad \operatorname{Ver}[T]$. Lema III.3.1., pág. 309.

Proposição 1.3 Seja $\mathcal{O}$ um conjunto aberto de $\Theta$ e suponha que para cada compacto $K \subset \mathcal{O}$, existe um compacto $\widetilde{K} \subset \mathcal{O}$ contendo $K$ e a $>0$ tais que

$$
F_{\widetilde{K}^{(}}(t, \dot{\xi}) \geq a \quad, \forall(t, \dot{\xi}) \in K \times P
$$

Então $\mathbb{D}$ é $\mathbb{G}^{s}$-globalmente hipoelíptico em $\mathcal{O} \times P$.

\section{Demonstração :}

$\triangleright \quad$ Para $c>0$ tomamos $c^{\prime}<c$ e $(t, \dot{\xi}) \in K \times P$. Escolhemos $s_{0} \in \widetilde{K}_{0}(t, \dot{\xi} ; 0)$ tal que

$$
B(t, \dot{\xi})-B\left(s_{0}, \dot{\xi}\right) \geq a .
$$

Observemos que o máximo de $B(\cdot, \dot{\xi})$ em $\widetilde{K}_{0}(t, \dot{\xi} ; 0)$ é $B(t, \dot{\xi})$. Assim, aplicando o Lema 4 para $K_{0}=\widetilde{K}_{0}(t, \dot{\xi} ; 0) \subset \widetilde{K}, B(s, \dot{\xi})$ e $\epsilon=\frac{\epsilon_{0}}{1+\rho}$ $\operatorname{com} \epsilon_{0}=\inf \left(1, \operatorname{dist}\left(K_{0}, \partial \Theta\right)\right)$, obtemos a existência de um caminho 
de integração linear por partes $\gamma_{t, s_{0}}$, juntando $t$ e $s_{0}$, contido numa vizinhança compacta $K^{\prime}$ de $\widetilde{K}$ contida em $\mathcal{O}$ sobre a qual

$$
B(s, \dot{\xi}) \leq B(t, \dot{\xi})+\frac{\epsilon_{0}}{1+\rho}, \quad \rho>0 .
$$

Além disso, existe $C>0$ tal que o comprimento de $\gamma_{t, s_{0}}$ é limitado por $C\left(\frac{1+\rho}{\epsilon_{0}}\right)^{n-1}$.

Seja $v \in \mathcal{C}^{\infty}(\mathcal{O})$. Integrando $d v$ sobre $\gamma_{t, s_{0}}$ obtemos

$$
-\int_{\gamma_{t, s_{0}}} d v(s)=v(t)-v\left(s_{0}\right)
$$

Portanto, multiplicando por $e^{-\rho B(t, \dot{\xi})}$, temos que:

$$
\begin{aligned}
e^{-\rho B(t, \dot{\xi})} v(t)= & e^{-\rho\left(B(t, \dot{\xi})-B\left(s_{0}, \dot{\xi}\right)\right)} e^{-\rho B\left(s_{0}, \dot{\xi}\right)} v\left(s_{0}\right) \\
& -\int_{\gamma_{t, s_{0}}} e^{-\rho(B(t, \dot{\xi})-B(s, \dot{\xi}))} e^{-\rho B(s, \dot{\xi})} d v(s) .
\end{aligned}
$$

Assim de (1.10) e (1.11):

$$
\begin{aligned}
e^{-\rho B(t, \dot{\xi})}|v(t)| \leq & e^{-\rho a} \sup _{s \in \widetilde{K}} e^{-\rho B(s, \dot{\xi})}|v(s)| \\
& +C\left(\frac{1+\rho}{\epsilon_{0}}\right)^{n-1} \sup _{s \in K^{\prime}} e^{-\rho B(s, \dot{\xi})}|d v(s)| .
\end{aligned}
$$

Multiplicando agora por $e^{c^{\prime} \rho^{1 / s}}$ obtemos

$$
\begin{aligned}
e^{c^{\prime} \rho^{1 / s}} e^{-\rho B(t, \dot{\xi})}|v(t)| \leq & e^{c^{\prime} \rho^{1 / s}-a \rho} \sup _{s \in \widetilde{K}} e^{-\rho B(s, \dot{\xi})}|v(s)| \\
& +C\left(\frac{1+\rho}{\epsilon_{0}}\right)^{n-1} e^{c^{\prime} \rho^{1 / s}} \sup _{s \in K^{\prime}} e^{-\rho B(s, \dot{\xi})}|d v(s)| .
\end{aligned}
$$

Como $e^{c^{\prime} \rho^{1 / s}-a \rho} \leq 1$ e $C\left(\frac{1+\rho}{\epsilon_{0}}\right)^{n-1} e^{c^{\prime} \rho^{1 / s}} \leq C_{1} e^{c \rho^{1 / s}}$ para alguma constante $C_{1}$ segue que

$e^{c^{\prime} \rho^{1 / s}} e^{-\rho B(t, \dot{\xi})}|v(t)| \leq \sup _{s \in \widetilde{K}} e^{-\rho B(s, \dot{\xi})}|v(s)|+C_{1} e^{c \rho^{1 / s}} \sup _{s \in K^{\prime}} e^{-\rho B(s, \dot{\xi})}|d v(s)|$. 
Vamos agora introduzir uma seqüência de funções obtidas de $F_{K}$ por processo iterativo. Para $\mu \geq 0, j \in \mathbf{N}, K$ subconjunto compacto de $\Theta$ e $(t, \dot{\xi}) \in K \times S^{m-1}$ definimos

$$
\begin{aligned}
& E_{K, \mu}^{0}(t, \dot{\xi})=F_{K}(t, \dot{\xi}), \\
& E_{K, \mu}^{j}(t, \dot{\xi})=F_{K}\left(t, \dot{\xi} ; \mu E_{K, \mu}^{j-1}(t, \dot{\xi})\right), j \geq 1, \\
& E_{K, \mu}(t, \dot{\xi})=\sup _{j \geq 0} E_{K, \mu}^{j}(t, \dot{\xi}) .
\end{aligned}
$$

Note que esta última função está bem definida, pois de (1.8)

$$
F_{K}(t, \dot{\xi}, r) \leq B(t, \dot{\xi})-\inf _{s \in K} B(s, \dot{\xi}) .
$$

Algumas propriedades das $E_{K, \mu}^{j}$ são:

$$
\begin{aligned}
& 0 \leq E_{K, \mu}^{0} \leq E_{K, \mu}^{1} \leq \cdots \\
& E_{K, \mu}^{0}(t, \dot{\xi})=0 \Rightarrow E_{K, \mu}^{j}(t, \dot{\xi})=0 \quad, \quad \forall j \geq 0 \\
& E_{K, \mu}^{j+1}(t, \dot{\xi})=E_{K, \mu}^{j}(t, \dot{\xi}) \Rightarrow E_{K, \mu}^{j^{\prime}}(t, \dot{\xi})=E_{K, \mu}^{j}(t, \dot{\xi}), \forall j^{\prime} \geq j .
\end{aligned}
$$

Definição 1.6 [M]. Sejam $\mathcal{O} \subset \Theta$ aberto e $P \subset S_{m-1}$ compacto. Dizemos que a função $B \in \mathcal{C}^{\infty}\left(\Theta \times S^{m-1}\right)$ satisfaz a condição $(R)$ em $\mathcal{O} \times P$ se, para cada conjunto compacto $K \subset \mathcal{O}$ existe um compacto $K^{\prime} \subset \mathcal{O}$ contendo $K$ e $\mu^{\prime} \geq 0, a>0$ tais que

$$
(t, \dot{\xi}) \in K \times P, \quad E_{K^{\prime}, \mu^{\prime}}(t, \dot{\xi}) \neq 0 \Rightarrow E_{K^{\prime}, \mu^{\prime}}(t, \dot{\xi}) \geq a .
$$

Observação 4 1. Se $B(t, \dot{\xi})$ não tivesse mínimo local em $\mathcal{O}$ e $K^{\prime}(t, \dot{\xi})$ tivesse um número finito de componentes então, pelo Lema 3 e (1.12), a condição (1.15) se reduziria para

$$
E_{K^{\prime}, \mu^{\prime}}(t, \dot{\xi}) \geq a \quad, \quad \forall(t, \dot{\xi}) \in K \times P .
$$


2. Se $B(t, \dot{\xi})$ só tivesse pontos críticos não degenerados em $\mathcal{O}$ para $\dot{\xi} \in P$ então $B$ satisfaria $(R)$ em $\mathcal{O} \times P$ se e só se $B(\cdot, \dot{\xi})$ não tivesse mínimo local em $\mathcal{O}$, para $\dot{\xi} \in P$. De fato, suponha que 0 é um ponto crítico não degenerado de $B(t, \dot{\xi})$ em $\mathcal{O}$ para $\dot{\xi} \in P$. Aplicando o lema de Morse podemos assumir que

$$
B(t, \dot{\xi})=B(0, \dot{\xi})+Q(t)
$$

com

$$
Q(t)=-t_{1}^{2}-\cdots-t_{l}^{2}+t_{l+1}^{2}+\cdots+t_{n}^{2}
$$

em uma vizinhança $U \times V$ de $\left(0, \dot{\xi}_{0}\right) \in \mathcal{O} \times P$, onde o número $l$ é o indice do ponto 0.

Seja $K$ uma vizinhança compacta de 0 . Pela condição $(R)$ existem $K^{\prime}$ e $a>0$ tal que $F_{K^{\prime}}(0, \dot{\xi}) \geq a$. Portanto $l \neq 0$ e assim 0 não é mínimo local. Reciprocamente, se 0 não é mínimo local, $l \neq 0$ e assim (1.16) implica que $F_{K^{\prime}}(0, \dot{\xi}) \geq a$.

3. Se $n=1$ e $d_{t} B=\frac{\partial B}{\partial t}=b$ não se anula em intervalos abertos não vazios contidos em $\Theta$, isto é a condição $(Q)$ de [T1], então $(R)$ se satisfaz em qualquer $\mathcal{O} \subset \Theta$ aberto se e só se b não muda de sinal de - para + em $\Theta$. Seja $\mathcal{O} \subset \Theta$ aberto. Se $B$ satisfaz $(R)$ em $\mathcal{O}$ então $B(t, \dot{\xi})$ não tem mínimo local em $\mathcal{O}$ e assim b não muda de sinal de - para + . Reciprocamente, suponha que b não muda de sinal de - para + em $\Theta$. Seja $\mathcal{O} \subset \Theta$ aberto. Dado $\left(t_{0}, \dot{\xi}\right) \in \mathcal{O} \times S^{m-1}$, existe uma vizinhança $U \times V$ dele e um conjunto compacto $K^{\prime} \subset \mathcal{O}$ tal que $F_{K}(t, \dot{\xi}) \geq a^{\prime}>0$ para $(t, \dot{\xi}) \in U \times V$. 
Proposição 1.4 Sejam $\mathcal{O} \subset \Theta$ e $P \subset S^{m-1}$ compacto. Se o operador $\mathbb{D}=d_{t}+d_{t} B\left(t, D_{x}\right)$ é $\mathbb{G}^{s}$-globalmente hipoelíptico em $\mathcal{O} \times P$ então a função $B$ satisfaz a condição $(R)$ em $\mathcal{O} \times P$.

Para a prova desta proposição precisamos do seguinte lema.

Lema $5[M]$. Sejam $K^{\prime}, K^{\prime \prime}$ subconjuntos compactos de $\mathcal{O}, K^{\prime} \subset$ int $K^{\prime \prime}$ e $P$ aberto em $S^{m-1}$. Então para cada $l \in \mathbb{N}$, existe $A_{l}>0$ tal que dados $\delta>0$ $e(t, \dot{\xi}) \in K^{\prime} \times P$, existe $v \in \mathcal{C}^{\infty}(\mathcal{O})$ com as seguintes propriedades:

1. $0 \leq v \leq 1, v(t)=1$, supp $v \subset K_{0}^{\prime \prime}(t, \dot{\xi} ; \delta)$

2. para $s \in K^{\prime} \cap \operatorname{supp} d v, B(s, \dot{\xi}) \geq B(t, \dot{\xi})+\delta / 4 e$ $\sum_{|\alpha| \leq l}\left|\partial_{t}^{\alpha} v(s)\right| \leq A_{l} / \delta^{l}$.

\section{Demonstração da proposição 1.4 :}

$\triangleright \quad$ Suponha que $B$ não satisfaz $(R)$ em $\mathcal{O} \times P$. Então existe um conjunto compacto $K \subset \mathcal{O}$ tal que, para cada compacto $K^{\prime \prime} \subset \mathcal{O}$ contendo $K$ e para cada $\mu \geq 0, a>0$ existem $(t, \dot{\xi}) \in K \times P$ com

$$
0<E_{K^{\prime \prime}, \mu}(t, \dot{\xi})<a
$$

Agora, pela definição de $E_{K^{\prime \prime}, \mu}$, existe $j \in \mathbb{Z}$, dependendo de $(t, \dot{\xi})$, tal que $E_{K^{\prime \prime}, \mu}^{j}(t, \dot{\xi})>\frac{1}{2} E_{K^{\prime \prime}, \mu}(t, \dot{\xi})$ e portanto disto e de (1.12) temos

$$
0<E_{K^{\prime \prime}, \mu}^{j}(t, \dot{\xi}) \leq E_{K^{\prime \prime}, \mu}^{j+1}(t, \dot{\xi})<E_{K^{\prime \prime}, \mu}(t, \dot{\xi})<2 E_{K^{\prime \prime}, \mu}^{j}(t, \dot{\xi})<2 a .
$$

Aplicando a Proposição 1.2 para $c>0$ e o conjunto compacto $K$ anterior, obtemos a existência de $c^{\prime}>0$ e $K^{\prime} \subset \mathcal{O}$ compacto, $m^{\prime} \in \mathbb{Z}^{+}$e 
$C>0$ tal que

$$
\begin{aligned}
& e^{c^{\prime} \rho^{1 / s}} \sup _{t \in K} e^{-\rho B(t, \dot{\xi})}|v(t)| \leq C\left\{\sup _{t \in K^{\prime}} e^{-\rho B(t, \dot{\xi})}|v(t)|\right. \\
&\left.+\sum_{|\alpha| \leq m^{\prime}} e^{c \rho^{1 / s}} \sup _{t \in K^{\prime}} e^{-\rho B(t, \dot{\xi})}\left|\partial_{t}^{\alpha} d v(t)\right|\right\}
\end{aligned}
$$

$\forall \rho>0, \quad \forall \dot{\xi} \in P, \quad \forall v \in \mathcal{C}^{\infty}(\Theta)$, além disso podemos supor $K^{\prime} \supset K$.

Agora escolhemos o $K^{\prime \prime}$ de (1.18) como se fosse uma vizinhança compacta de $K^{\prime}$ contida em $\mathcal{O}$ ( $\mu$ e $a$ serão escolhidos depois).

Assim, aplicando o Lema 5 para os compactos anteriores e com

$$
\delta=\mu E_{K^{\prime \prime}, \mu}^{j}(t, \dot{\xi})
$$

obtemos a existência de uma função $v \in \mathcal{C}^{\infty}(\mathcal{O})$ com as propriedades 1. e 2 ..

Com esta $v$ em particular, (1.19) se verifica. Portanto, levando em conta as propriedades do Lema 5, temos:

$$
\begin{aligned}
e^{c^{\prime} \rho^{1 / s}} e^{-\rho B(t, \dot{\xi})} \leq C & \left\{\sup _{s \in K_{0}^{\prime \prime}(t, \dot{\xi} ; \delta)} e^{-\rho B(s, \dot{\xi})}\right. \\
& \left.+\frac{A_{m^{\prime}}}{\delta^{m^{\prime}}} e^{c \rho^{1 / s}} \sup _{s \in K^{\prime} \cap \text { supp } d v} e^{-\rho B(s, \dot{\xi})}\right\} \quad \forall \rho \geq 0 .
\end{aligned}
$$

Assim,

$$
\begin{aligned}
e^{c^{\prime} \rho^{1 / s}} \leq C & \left\{\sup _{s \in K_{0}^{\prime \prime}(t, \dot{\xi} ; \delta)} e^{-\rho(B(s, \dot{\xi})-B(t, \dot{\xi}))}\right. \\
& \left.+\frac{A_{m^{\prime}}}{\delta m^{\prime}} e^{c \rho^{1 / s}} \sup _{s \in K^{\prime} \cap \operatorname{supp} d v} e^{-\rho(B(s, \dot{\xi})-B(t, \dot{\xi}))}\right\} \quad \forall \rho \geq 0 .
\end{aligned}
$$


Portanto, pela definição de $F_{K^{\prime \prime}}$, pelo Lema 5 parte 2. e (1.20), segue que

$$
\begin{aligned}
e^{c^{\prime} \rho^{1 / s}} & \leq C\left\{e^{\rho F_{K^{\prime \prime}}(t, \dot{\xi} ; \delta)}+\frac{A_{m^{\prime}}}{\delta^{\prime}} e^{c \rho^{1 / s}} e^{-\rho \delta / 4}\right\} \\
& =C\left\{e^{\rho F_{K^{\prime \prime}}\left(t, \dot{\xi} ; \mu E_{K^{\prime \prime}, \mu}^{j}(t, \dot{\xi})\right)}+\frac{A_{m^{\prime}}}{\left(\mu E_{K^{\prime \prime}, \mu}^{j}(t, \dot{\xi})\right)^{m^{\prime}}} e^{c \rho^{1 / s}} e^{-\rho \mu E_{K^{\prime \prime}, \mu}^{j}(t, \dot{\xi}) / 4}\right\} .
\end{aligned}
$$

Escolhendo $\mu=16 \mathrm{~m}^{\prime} \mathrm{c}$ e levando em conta (1.18) obtemos que:

$$
\begin{aligned}
e^{c^{\prime} \rho^{1 / s}} & \leq C\left\{e^{\rho E_{K^{\prime \prime}, \mu}^{j+1}(t, \dot{\xi})}+\frac{A_{m^{\prime}}}{\left(16 m^{\prime} c E_{K^{\prime \prime}, \mu}^{j}(t, \dot{\xi})\right)^{m^{\prime}}} e^{c \rho^{1 / s}} e^{-4 m^{\prime} c \rho E_{K^{\prime \prime}, \mu}^{j}(t, \dot{\xi})}\right\} \\
& \leq C\left\{e^{2 \rho E_{K^{\prime \prime}, \mu}^{j}(t, \dot{\xi})}+\frac{A_{m^{\prime}}}{\left(16 m^{\prime} c E_{K^{\prime \prime}, \mu}^{j}(t, \dot{\xi})\right)^{m^{\prime}}} e^{c \rho^{1 / s}} e^{-4 m^{\prime} c \rho E_{K^{\prime \prime}, \mu}^{j}(t, \dot{\xi})}\right\} .
\end{aligned}
$$

Resumindo e mudando de notações, concluímos quẹ para algum $m^{\prime}>0$ e para cada $a>0$, existem $a^{\prime}>0, a^{\prime}<a$ tal que:

$$
e^{c^{\prime} \rho^{1 / s}} \leq C_{1}\left\{e^{2 a^{\prime} \rho}+e^{c \rho^{1 / s}}\left(\frac{e^{-4 a^{\prime} c \rho}}{a^{\prime}}\right)^{m^{\prime}}\right\}, \quad \forall \rho \geq 0,
$$

e alguma constante $C_{1}>0$.

Agora, se escolhemos

$$
a^{\prime} \doteq g(\rho) \doteq \frac{\ln \left(\frac{1}{2 C} e^{c^{\prime} \rho^{1 / s}}\right)}{2 \rho}
$$

temos que

$$
e^{2 \rho g(\rho)}=\frac{1}{2 C} e^{c^{\prime} \rho^{1 / s}} .
$$

Com isto a desigualdade (1.21) fica como

$$
\frac{1}{2} e^{c^{\prime} \rho^{1 / s}} \leq C_{1} e^{c \rho^{1 / s}}\left(\frac{e^{-4 c \rho g(\rho)}}{g(\rho)}\right)^{m^{\prime}}, \quad \forall \rho \geq 0 .
$$

Observando que

$$
\lim _{\rho \rightarrow \infty} g(\rho) \rho^{1-1 / s}=\frac{c^{\prime}}{2}
$$


concluímos que para $\rho$ grande, $g$ se comporta como $\rho^{1 / s-1}$ o qual nos produz uma contradição com (1.22).

A seguir damos uma condição evidentemente necessária para que $\mathbb{D}$ seja $\mathbb{G}^{s}$-Hipoelíptico em $\Theta \times P$.

Lema 6 Se $\mathbb{D}=D_{t}+d_{t} B\left(t, D_{x}\right)$ é $\mathbb{G}^{s}$-Hipoelíptico em $\Theta \times P$ então para cada conjunto aberto $\mathcal{O} \subset \Theta$ e para cada $\dot{\xi} \in P, d_{t} B(\cdot, \dot{\xi})$ não se anula identicamente em $\mathcal{O}$.

\section{Demonstração :}

$\triangleright \quad$ Suponha que para algum conjunto $\mathcal{O} \subset \Theta$ e $\dot{\xi} \in P$ temos que $\left.d_{t} B\right|_{\mathcal{O}_{\times\{\dot{\xi}\}}}=0$. Aplicando a desigualdade (1.4) com $K$ reduzido a qualquer ponto de $\mathcal{O}$ e $v=1$ em $\mathcal{O}$ nos conduz a um absurdo. Portanto, pela Proposição $1.2 \mathbb{D}$ não é $\mathbb{G}^{s}$-Globalmente hipoelíptico em $\mathcal{O} \times P$. Assim, $\mathbb{D}$ não é $\mathbb{G}^{s}$-Hipoelíptico em $\Theta \times P$.

Teorema $1 O$ operador $\mathbb{D}=D_{t}+d_{t} B\left(t, D_{x}\right)$ é $\mathbb{G}^{s}$-Hipoelíptico em $\Theta \times P$ se e só se para cada conjunto aberto $\mathcal{O} \subset \Theta$, a função $B$ satisfaz a condição $(R)$ em $\mathcal{O} \times P$ e d $d_{t} B(\cdot, \dot{\xi})$ não é identicamente zero em $\mathcal{O}$, para cada $\dot{\xi} \in P$.

Lema 7 Sejam $K \subset K^{\prime}$ subconjuntos compactos de $\mathcal{O} \subset \Theta,(t, \dot{\xi}) \in K \times$ $S^{m-1}$ e $\rho, \mu^{\prime} \in \mathbf{R}_{+}, j \in \mathbb{N}$ e $c>0$. Suponha que

$$
\frac{1}{c^{\prime}} E_{K^{\prime}, \mu^{\prime}}^{j-1}(t, \dot{\xi}) \leq \rho^{\frac{1-s}{s}}<\frac{1}{c^{\prime}} E_{K^{\prime}, \mu^{\prime}}^{j}(t, \dot{\xi}), \quad c^{\prime}\left(\mu^{\prime}+1\right)<c .
$$

Então existem $C>0, \epsilon_{0}>0$ dependendo só de $K, K^{\prime}$ tais que

$$
\begin{aligned}
e^{c^{\prime} \rho^{1 / s}} e^{-\rho B(t, \dot{\xi})}|v(t)| & \leq \sup _{s \in K^{\prime}} e^{-\rho B(s, \dot{\xi})}|v(s)| \\
& +C e^{c \rho^{1 / s}} \sup _{s \in K^{\prime \prime}} e^{-\rho B(s, \dot{\xi})}|d v(s)|, \quad \forall v \in \mathcal{C}^{\infty}(\mathcal{O}) e t \in K,
\end{aligned}
$$

onde $K^{\prime \prime}$ é uma $\epsilon_{0}$-vizinhança de $K^{\prime}$. 


\section{Demonstração :}

$\triangleright \quad$ Seja $v \in \mathcal{C}^{\infty}(\mathcal{O})$. Escolhemos $s_{j} \in K_{0}^{\prime}\left(t, \dot{\xi}_{;} \mu^{\prime} E_{K^{\prime}, \mu^{\prime}}^{j-1}(t, \dot{\xi})\right)$ tal que

$$
B(t, \dot{\xi})-B\left(s_{j}, \dot{\xi}\right)=E_{K^{\prime}, \mu^{\prime}}^{j}(t, \dot{\xi}) .
$$

Aplicando o Lema 4 para $K_{0}^{\prime}\left(t, \dot{\xi} ; \mu^{\prime} E_{K^{\prime}, \mu^{\prime}}^{j-1}(t, \dot{\xi})\right), B(s, \dot{\xi})$ e $\epsilon_{0} /(\rho+1)$, onde $\epsilon_{0}$ é escolhido de tal forma que a vizinhança $K^{\prime}$ da ordem $\epsilon_{0}$ de $K^{\prime}$ esta contida em $\mathcal{O}$, obtemos um caminho de integração $\gamma_{t, s_{j}}$, contido em $\mathcal{O}$, tal que

$$
\sup _{s \in \gamma_{t, s_{j}}} B(s, \dot{\xi}) \leq \sup _{s \in K_{0}^{\prime}\left(t, \dot{\xi} ; \mu^{\prime} E_{K^{\prime}, \mu^{\prime}}^{j-1}(t, \dot{\xi})\right)} B(s, \dot{\xi})+\epsilon_{0} /(\rho+1)
$$

e o comprimento de $\gamma_{t, s_{j}}$ é limitado por $C\left(\frac{\rho+1}{\epsilon_{0}}\right)^{n-1}$, onde $C$ depende só de $K$ e $K^{\prime}$.

Integrando sobre $\gamma_{t, s_{j}}$ temos

$$
\begin{aligned}
e^{-\rho B(t, \dot{\xi})} v(t)= & e^{-\rho\left(B(t, \dot{\xi})-B\left(s_{j}, \dot{\xi}\right)\right)} e^{-\rho B\left(s_{j}, \dot{\xi}\right)} v\left(s_{j}\right) \\
& -\int_{\gamma_{t, s_{j}}} e^{-\rho(B(t, \dot{\xi})-B(s, \dot{\xi}))} e^{-\rho B(s, \dot{\xi})} d v(s) .
\end{aligned}
$$

Portanto, de (1.25) e (1.26),

$$
\begin{aligned}
e^{-\rho B(t, \dot{\xi})}|v(t)| & \leq e^{-\rho E_{K^{\prime} \mu^{\prime}}^{j}(t, \dot{\xi})} e^{-\rho B\left(s_{j}, \dot{\xi}\right)}\left|v\left(s_{j}\right)\right| \\
+ & C\left(\frac{\rho+1}{\epsilon_{0}}\right)^{n-1} e^{\rho\left(\mu^{\prime} E_{K^{\prime} \mu^{\prime}}^{j-1}(t, \dot{\xi})+\epsilon_{0} /(\rho+1)\right)} \sup _{s \in K^{\prime \prime}} e^{-\rho B(s, \dot{\xi})}|d v(s)| .
\end{aligned}
$$

Assim, de (1.23),

$$
\begin{aligned}
e^{-\rho B(t, \dot{\xi})}|v(t)| \leq & e^{-c^{\prime} \rho^{1 / s}} \sup _{s \in K^{\prime}} e^{-\rho B(s, \dot{\xi})}|v(s)| \\
& +C\left(\frac{\rho+1}{\epsilon_{0}}\right)^{n-1} e^{\rho\left(\mu^{\prime} c^{\prime} \rho^{\frac{1-s}{s}}+\epsilon_{0} /(\rho+1)\right)} \sup _{s \in K^{\prime \prime}} e^{-\rho B(s, \dot{\xi})}|d v(s)| .
\end{aligned}
$$




$$
\begin{aligned}
& \text { Logo } \\
& \begin{array}{l}
e^{c^{\prime} \rho^{1 / s}} e^{-\rho B(t, \dot{\xi})} \\
|v(t)| \leq \sup _{s \in K^{\prime}} e^{-\rho B(s, \dot{\xi})}|v(s)| \\
\quad+C\left(\frac{\rho+1}{\epsilon_{0}}\right)^{n-1} e^{\mu^{\prime} c^{\prime} \rho^{1 / s}+\epsilon_{0}} e^{c^{\prime} \rho^{1 / s}} \sup _{s \in K^{\prime \prime}} e^{-\rho B(s, \dot{\xi})}|d v(s)| \\
\quad=\sup _{s \in K^{\prime}} e^{-\rho B(s, \dot{\xi})}|v(s)|+C^{\prime} \rho^{n-1} e^{c^{\prime}\left(\mu^{\prime}+1\right) \rho^{1 / s}} \sup _{s \in K^{\prime \prime}} e^{-\rho B(s, \dot{\xi})}|d v(s)| \\
\leq \sup _{s \in K^{\prime}} e^{-\rho B(s, \dot{\xi})}|v(s)|+C^{\prime} e^{c \rho^{1 / s}} \sup _{s \in K^{\prime \prime}} e^{-\rho B(s, \dot{\xi})}|d v(s)|,
\end{array}
\end{aligned}
$$

para alguma constante $C^{\prime}>0$.

\section{Demonstração do teorema 1 :}

$\triangleright \quad$ A Proposição 1.4 e o Lema 6 mostram que a condição é necessária para a $\mathbb{G}^{s}$-hipoelipticidade. Vamos mostrar que esta é suficiente. Provaremos que dado $\mathcal{O}$ existem $K^{\prime} \subset \mathcal{O}, m^{\prime} \in \mathrm{N}, c^{\prime}>0$ e $C>0$ tais que (1.4) se verifica.

Pela condição $(R)$ existem $K^{\prime}$ compacto em $\mathcal{O}, \mu^{\prime} \geq 0$ e $a>0$ tal que (1.15) se satisfaz.

Seja $(t, \dot{\xi}) \in K \times P$ tal que $E_{K^{\prime}, \mu^{\prime}}(t, \dot{\xi}) \neq 0$. Portanto, existe um inteiro $k=k(t, \dot{\xi})$ e $a^{\prime}>0$ tal que

$$
E_{K^{\prime}, \mu^{\prime}}^{k}(t, \dot{\xi}) \geq a^{\prime} / 2
$$

Dado $\rho>0$, com $c^{\prime}\left(\mu^{\prime}+1\right)<c$, temos uma das três possibilidades:

1. $\rho^{\frac{1-s}{s}}<\frac{1}{c^{\prime}} E_{K^{\prime}, \mu^{\prime}}^{0}(t, \dot{\xi})$,

2. $\frac{1}{c^{\prime}} E_{K^{\prime}, \mu^{\prime}}^{j-1}(t, \dot{\xi}) \leq \rho^{\frac{1-s}{s}}<\frac{1}{c^{\prime}} E_{K^{\prime}, \mu^{\prime}}^{j}(t, \dot{\xi})$, para algum $j \leq k$,

3. $\frac{1}{c^{\prime}} E_{K^{\prime}, \mu^{\prime}}^{k}(t, \dot{\xi}) \leq \rho^{\frac{1-\varepsilon}{s}}$.

No caso 1., escolhemos $s_{0} \in K_{0}^{\prime}(t, \dot{\xi} ; 0)$ tal que

$$
B(t, \dot{\xi})-B\left(s_{0}, \dot{\xi}\right)=E_{K^{\prime}, \mu^{\prime}}^{0}(t, \dot{\xi})=F_{K^{\prime}}(t, \dot{\xi}) .
$$


Então, pelo Lema 4, existe um caminho de integração linear por partes $\gamma_{t, s_{0}}$, contido numa vizinhança compacta $K^{\prime \prime}$ de $K^{\prime}$ contida em $\mathcal{O}$ sobre a qual

$$
B(s, \dot{\xi}) \leq B(t, \dot{\xi})+\frac{1}{\rho+1}
$$

e o $\gamma_{t, s_{0}}$ é limitado por $C(\rho+1)^{n-1}$.

Dada $v \in \mathcal{C}^{\infty}(\mathcal{O})$, integrando sobre $\gamma_{t, s_{0}}$ obtemos

$$
\begin{aligned}
e^{-\rho B(t, \dot{\xi})} v(t)= & e^{-\rho\left(B(t, \dot{\xi})-B\left(s_{0}, \dot{\xi}\right)\right)} e^{-\rho B\left(s_{0}, \dot{\xi}\right)} v\left(s_{0}\right) \\
& -\int_{\gamma_{t, s_{0}}} e^{-\rho(B(t, \dot{\xi})-B(s, \dot{\xi}))} e^{-\rho B(s, \dot{\xi})} d v(s) .
\end{aligned}
$$

De (1.27) e (1.28) segue que

$$
\begin{aligned}
e^{-\rho B(t, \dot{\xi})}|v(t)| \leq & e^{-\rho F_{K^{\prime}}(t, \dot{\xi})} \sup _{s \in K^{\prime}} e^{-\rho B(s, \dot{\xi})}|v(s)| \\
& +C(\rho+1)^{n-1} \sup _{s \in K^{\prime \prime}} e^{-\rho B(s, \dot{\xi})}|d v(s)| \\
\leq & e^{-c^{\prime} \rho^{1 / s}} \sup _{s \in K^{\prime}} e^{-\rho B(s, \dot{\xi})}|v(s)| \\
& +C(\rho+1)^{n-1} \sup _{s \in K^{\prime \prime}} e^{-\rho B(s, \dot{\xi})}|d v(s)| .
\end{aligned}
$$

Como $c-c^{\prime}>0$ implica $(\rho+1)^{n-1} e^{c^{\prime} \rho^{1 / s}}<e^{c \rho^{1 / s}}$ para $\rho>0$ grande, temos que

$$
\begin{aligned}
e^{c^{\prime} \rho^{1 / s}} e^{-\rho B(t, \dot{\xi})}|v(t)| \leq & \sup _{s \in K^{\prime}} e^{-\rho B(s, \dot{\xi})}|v(s)| \\
& +C(\rho+1)^{n-1} e^{c^{\prime} \rho^{1 / s}} \sup _{s \in K^{\prime \prime}} e^{-\rho B(s, \dot{\xi})}|d v(s)| \\
\leq & \sup _{s \in K^{\prime}} e^{-\rho B(s, \dot{\xi})}|v(s)| \\
& +C e^{c \rho^{1 / s}} \sup _{s \in K^{\prime \prime}} e^{-\rho B(s, \dot{\xi})}|d v(s)| .
\end{aligned}
$$

O caso 2. segue do lema 7. 
No caso 3. temos

$$
\rho<\left(\frac{2 c^{\prime}}{a^{\prime}}\right)^{\frac{s}{s-1}} \doteq A,
$$

onde $A$ não depende de $(t, \dot{\xi}) \in K \times P$. Para estes $\rho$ obviamente existe $C>0$ tal que:

$$
\begin{aligned}
e^{\rho^{1 / s}} e^{-\rho B(t, \dot{\xi})}|v(t)| \leq C & \left\{\sup _{s \in K^{\prime}} e^{-\rho B(s, \dot{\xi})}|v(s)|\right. \\
& \left.+e^{c \rho^{1 / s}} \sup _{s \in K^{\prime}} e^{-\rho B(s, \dot{\xi})}|d v(s)|\right\} .
\end{aligned}
$$

Até agora, temos (1.29) válido para todo $(t, \dot{\xi}) \in K \times P, \rho>0$ tal que $E_{K^{\prime}, \mu^{\prime}}(t, \dot{\xi}) \neq 0$. Usamos agora o fato que $t \mapsto d_{t} B(t, \dot{\xi})$ não é identicamente zero em qualquer conjunto aberto. Portanto, dado $(t, \dot{\xi})$ com $E_{K^{\prime}, \mu^{\prime}}(t, \dot{\xi})=0$ existe uma seqüência $\left\{t_{j}\right\}, t_{j} \rightarrow t, \operatorname{com} E_{K^{\prime}, \mu^{\prime}}\left(t_{j}, \dot{\xi}\right) \neq 0$. Assim obtemos por continuidade que (1.29) é verdadeira para qualquer $(t, \dot{\xi}) \in K \times P$ e $\rho>0$. 


\section{Capítulo 2}

\section{Hipoelipticidade Gevrey quando a Estrutura é Tubular}

\subsection{Classes de Gevrey}

Definimos as classes de Gevrey de índice $s \geq 1$ e amplitude $h>0$ sobre o fecho de abertos $U \subset \subset \mathbf{R}^{N}$ com fronteira $\mathcal{C}^{\infty}$ como sendo subconjuntos

$$
G^{s, h}(\bar{U}) \subset \mathcal{C}^{\infty}(\bar{U})
$$

constituídos pelas funções $f$ infinitamente diferenciáveis em alguma vizinhança aberta de $\bar{U}$, a valores complexos, que satisfazem estimativas do tipo

$$
\left|D^{\alpha}[f](y)\right| \leq C h^{|\alpha|}(|\alpha| !)^{s}: \quad \forall y \in \bar{U}, \quad \forall \alpha \in \mathbf{Z}_{+}^{N}
$$

para alguma constante $C>0$. Na estimativa acima, usamos a notações

$$
D=\left(D_{1}, \cdots, D_{N}\right) \quad, \quad D_{j}=\frac{1}{i} \frac{\partial}{\partial y_{j}},
$$

$D^{\alpha}=D_{1}^{\alpha_{1}} \cdots D_{N}^{\alpha_{N}}$ e $|\alpha|=\alpha_{1}+\cdots+\alpha_{N}$. Cada classe $G^{s, h}(\bar{U})$ é um subespaço vetorial de $\mathcal{C}^{\infty}(\bar{U})$ completo em relação à norma definida pelo ínfimo das 
constantes $C$ na estimativa (2.1), a saber,

$$
\|f\|_{s, h, \bar{U}}=\sup \left\{\frac{\left|D^{\alpha}[f](y)\right|}{h^{|\alpha|}(|\alpha| !)^{s}}: y \in \bar{U}, \alpha \in \mathbf{Z}_{+}^{N}\right\} .
$$

Como exemplo de tais classes destacamos $G^{1, h}(\bar{U})$ que corresponde ao espaço das funções $f=f(y)$ analíticas reais em $\bar{U}$ que possuem extensões $f^{\#}=f^{\#}(z)$ holomorfas em $U^{\#}=\left\{z \in \mathbb{C}^{N}: \operatorname{dist}(z, \bar{U})<h^{-1}\right\}$.

$\mathrm{O}$ produto de funções ponto a ponto geralmente altera a amplitude das classes de Gevrey. Se $f$ e $g$ são funções respectivamente em $G^{s, h_{f}}(\bar{U})$ e $G^{s, h_{g}}(\bar{U})$ então podemos garantir que $f \cdot g$ pertence a $G^{s, 2 \max \left\{h_{f}, h_{g}\right\}}(\bar{U})$ e

$$
\|f \cdot g\|_{s, 2 \max \left\{h_{f}, h_{g}\right\}, \bar{U}} \leq\|f\|_{s, h_{f}, \bar{U}}\|g\|_{s, h_{g}, \bar{U}} .
$$

As derivações também alteram a amplitude das classes de Gevrey e, por tanto, não definem endomorfismos nestas classes. Se $f \in G^{s, h}(\bar{U})$ e se $\beta \in$ $\mathbf{Z}_{+}^{N} \backslash\{0\}$ então podemos garantir que $D^{\beta}[f] \in G^{s, 2^{s|\beta|} h_{f}}(\bar{U})$ com

$$
\left\|\left.D^{\beta}[f]\right|_{s, 2 s|\beta| h_{f}, \bar{U}} \leq 2^{s(|\beta|-1) !} h_{f}^{|\beta|}\right\| f \|_{s, h_{f}, \bar{U}} .
$$

Segue da estimativa (2.1) que as inclusões

$$
G^{s, h}(\bar{U}) \hookrightarrow G^{s, k}(\bar{U}), \quad h \leq k
$$

são contínuas com norma $\leq 1$.

Afirmação 2.1.1 As inclusões (2.4) são compactas se $h<k$.

A compacidade das inclusões (2.4) garante que os espaços

$$
G^{s}(\bar{U})=\bigcup_{h>0} G^{s, h}(\bar{U})
$$

sejam de Hausdorff com a topologia mais fina, de espaço localmente convexo, que torna tais inclusões contínuas. Mais ainda, os conjuntos limitados 
nesta topologia de limite indutivo são necessariamente limitados em alguma classe de amplitude $h>0$. Com isto, a convergência em conjuntos limitados equivale à convergência em alguma classe de amplitude $h>0$ fixada.

$\mathrm{O}$ espaço das classes Gevrey de índice $s \geq 1$ sobre um aberto $V \subset U$ é dado pelo limite indutivo

$$
G^{s}(V)=\lim _{\overline{V_{0}} \nearrow V} G^{s}\left(\overline{V_{0}}\right)
$$

É rotineiro estender a noção de classes Gevrey de índice $s \geq 1$ a uma variedade analítica real.

Segue de (2.2) e (2.3) que qualquer operador do tipo

$$
L=L(y D)=\sum_{|\beta| \leq m} a_{\beta}(y) D^{\beta}, a_{\beta} \in G^{s}(\bar{U})
$$

define endomorfismos contínuos em $G^{s}(\bar{U})$.

\subsection{Estruturas localmente integráveis e seu complexo. Estruturas tubulares e estru- turas hipocomplexas}

Seja $\Omega$ uma variedade diferenciável de classe $\mathcal{C}^{\infty}$, paracompacta, de dimensão $N$ e assumimos sobre $\Omega$ uma estrutura localmente integrável $\mathcal{V}$ de dimensão $n$. Assim $\mathcal{V}$ é um subfibrado vetorial (complexo) de $\mathbb{C} T \Omega$ de posto $n$ tal que seu fibrado ortogonal $\mathrm{T}^{\prime} \subset \mathbb{C T}^{*} \Omega$ é localmente gerado pelos diferenciais de $m \doteq N-n$ funções $\mathcal{C}^{\infty}$.

Fixada uma origem $O$ na variedade é possível considerar, (cf. [T2], Sec.I.7), um sistema de coordenadas locais

$$
(U,(x, t))=\left(U,\left(x_{1}, \ldots, x_{m}, t_{1}, \ldots, t_{n}\right)\right)
$$


centrado na origem na qual

$$
Z_{k}(x, t)=x_{k}+i \Phi_{k}(x, t), k=1, \ldots, m
$$

$\operatorname{com} \Phi=\left(\Phi_{1}, \ldots, \Phi_{m}\right)$ infinitamente diferenciável em $U$, a valores em $\mathbf{R}^{m}$, satisfazendo

$$
\Phi(0,0)=0, \Phi_{x}(0,0)=0,\left\|\Phi_{x}\right\|<\frac{1}{2} \text { em } U
$$

tais que os campos

$$
\mathrm{L}_{j}=\frac{\partial}{\partial t_{j}}+i \sum_{k=1}^{m} \lambda_{j k}(x, t) \frac{\partial}{\partial x_{k}} j=1, \cdots, n
$$

sejam geradores de $\mathcal{V}$ em $U$, onde

$$
\left(\lambda_{j k}\right)=-i \sum_{l=1}^{m} \frac{\partial Z_{l}}{\partial t_{j}} \mu_{l k} \operatorname{com}\left(\mu_{k l}\right)=\left[\left(\frac{\partial Z_{k}}{\partial x_{l}}\right)^{-1}\right]^{t} .
$$

Introduzindo os campos

$$
M_{k}=\sum_{l=1}^{m} \mu_{k l}(x, t) \frac{\partial}{\partial x_{l}}, k=1, \cdots, m
$$

segue que

$$
(\mathrm{M}, \mathrm{L})=\left(\mathrm{M}_{1}, \ldots, \mathrm{M}_{m}, \mathrm{~L}_{1}, \ldots, \mathrm{L}_{n}\right)
$$

constitui uma base de $\mathbb{C T} \Omega$ sobre $U$ dual de $\left(d Z_{1}, \ldots, d Z_{m}, d t_{1}, \ldots, d t_{n}\right)$. Os $\mathrm{M}_{k}$ e $\mathrm{L}_{j}$ são tais que

$$
\mathrm{M}_{k} t_{j}=0, \mathrm{~L}_{j} Z_{k}=0, \mathrm{M}_{k} Z_{k^{\prime}}=\delta_{k k^{\prime}}, \mathrm{L}_{j} t_{j^{\prime}}=\delta_{j j^{\prime}} .
$$

Aqui $\delta$ é a função delta de Kronecker.

Para tais estruturas a derivada exterior em $U$ define, após passagem ao quociente, um complexo de operadores diferenciais sobre $U$ no qual o primeiro operador se escreve como,

$$
\mathcal{C}^{\infty}(U) \stackrel{\mathrm{d}_{0}^{\prime}}{\longrightarrow} \mathcal{C}^{\infty}\left(U, \Lambda^{1}\left(\mathbb{C} \mathrm{T}^{*} U / \mathrm{T}^{\prime}\right)\right)
$$


Como $\left.\left(\mathbb{C} T^{*} U / \mathrm{T}^{\prime}\right)\right|_{U}$ pode ser identificado com o subfibrado vetorial de $\mathbb{C T}^{*} U$ gerado pelas formas $\left\{d t_{1}, \ldots, d t_{n}\right\}$, podemos identificar, para todo $V \subset U$ aberto, o espaço das seções $\mathcal{C}^{\infty}\left(V, \Lambda\left(\mathbb{C} T^{*} U / \mathrm{T}^{\prime}\right)\right)$ com o espaço $\mathcal{C}^{\infty}\left(U, \Lambda^{1}\right)$ das formas diferenciais da forma

$$
f=\sum_{j=1}^{n} f_{j}(x, t) d t_{j} .
$$

Então o complexo em questão se realiza, em dimensão zero e sobre $V$, da seguinte forma:

$$
\mathrm{L}: \mathcal{C}^{\infty}(V) \longrightarrow \mathcal{C}^{\infty}\left(V, \Lambda^{1}\right)
$$

onde

$$
\mathrm{L} u=\sum_{j=1}^{n} \mathrm{~L}_{j} u(x, t) d t_{j} .
$$

Definição 2.1 Uma estrutura localmente integrável é chamada de estrutura tubular se existem um sistema de coordenadas locais $(U,(x, t))$ e $Z$ como em (2.5) com $\operatorname{Im} Z$ independente de $x$.

Existe uma definição intrínseca deste conceito (cf. [T1], definição VI.9.2).

No caso em que a estrutura é tubular sobre $B \times \Theta, B \times \Theta \subset \mathbf{R}^{1+n}$ aberto não vazio $(m=1)$ podemos escrever (cf. [T2], Sec. I.12)

$$
Z(x, t)=x+i \Phi(t)
$$

onde os $\Phi$ são funções $\mathcal{C}^{\infty}$ em $\Theta$ e a valores reais, e

$$
\mathrm{L}_{j}=\frac{\partial}{\partial t_{j}}-i \frac{\partial \Phi}{\partial t_{j}} \frac{\partial}{\partial x}, \quad j=1, \ldots, n .
$$

Observe que $L$ pode ser escrito como

$$
\mathrm{L}=d_{t}-i d \Phi \wedge \frac{\partial}{\partial x} .
$$


Quando a aplicação

$$
\Phi=\left(\Phi_{1}, \ldots, \Phi_{m}\right): \Theta \longrightarrow \mathbf{R}^{m}
$$

é de classe $\mathcal{C}^{w}$ dizemos que a estrutura tubular é analítica.

Definição 2.2 Seja $\mathcal{V}$ uma estrutura localmente integrável sobre $\Omega$. $\mathcal{V}$ é dita hipocomplexa no ponto $P$ se vale a seguinte propriedade:

Qualquer que seja $U_{0} \subset U$ vizinhança de $P$, dada $u \in \mathcal{D}^{\prime}\left(U_{0}\right)$ satisfazendo $L u=0$ sobre $U_{0}$ existe uma função $H$ holomorfa numa vizinhança de $Z(P)$ em $\mathbb{C}^{m}$ tal que $u=H \circ Z$ numa vizinhança de $P$.

Proposição 2.1 Sejam $\mathcal{V}, P, U$ e $Z$ como na definição 2.2 e suponhamos $m=1$. Entâo $\mathcal{V}$ é hipocomplexa em $P$ se e somente se $Z$ é aberta em $P$ (isto é, $Z$ leva vizinhança de $P$ em $U$ em vizinhanças de $Z(P)$ em $\left.\mathbb{C}^{1}\right)$.

\section{Demonstração :}

$\triangleright \quad$ Ver [T2]. Corolário III.5.3.

Definição 2.3 Uma estrutura localmente integrável $\mathcal{V}$ sobre $\Omega$ é dita hipocomplexa se ela for hipocomplexa em todo ponto $P$ de $\Omega$.

\subsection{As classes $G_{m L}^{s}$}

Mantemos as notações introduzidas na seção 2.2. Dados $V \subset \subset U$ um aberto com fronteira $\mathcal{C}^{\infty}, s \geq 1$ e $h>0$ dizemos que $u \in \mathrm{G}_{\mathrm{ML}}^{s, h}(V)$ se $u \in$ $\mathcal{C}^{\infty}(\bar{V})$ e se existe $C>0$ tal que

$$
\sup _{\bar{V}}\left|\mathrm{M}^{\alpha_{1}} \mathrm{~L}^{\alpha_{2}} u\right| \leq \mathrm{const} \cdot h^{\left|\alpha_{1}\right|+\left|\alpha_{2}\right|}\left(\left|\alpha_{1}\right|+\left|\alpha_{2}\right|\right) !^{s}, \forall\left(\alpha_{1}, \alpha_{2}\right) \in \mathrm{Z}_{+}^{m+n} .
$$

Como em $[C]$, cada $\mathrm{G}_{\mathrm{mL}}^{s, h}(V)$ é um espaço de Banach em relação à norma 
definida pelo ínfimo das constantes em (2.7), a saber,

$$
\|u\|_{s, h, V}=\sup \left\{\frac{\left|\mathrm{M}^{\alpha_{1}} \mathrm{~L}^{\alpha_{2}} u(x, t)\right|}{h^{\left|\alpha_{1}\right|+\left|\alpha_{2}\right|}\left(\left|\alpha_{1}\right|+\left|\alpha_{2}\right|\right) ! s}:(x, t) \in \bar{V},\left(\alpha_{1}, \alpha_{2}\right) \in \mathrm{Z}_{+}^{m+n}\right\},
$$

e as inclusões

$$
\mathrm{G}_{\mathrm{ML}}^{s, h}(V) \hookrightarrow \mathrm{G}_{\mathrm{ML}}^{s, h_{+}}(V), h<h_{+}
$$

são compactas. Explicitando,

$$
\mathrm{G}_{\mathrm{ML}}^{s, h}(V)=\left\{u \in \mathcal{C}^{\infty} \bar{V}:\|u\|_{s, h, V}<\infty\right\} .
$$

Também como em [C], os espaços

$$
\mathrm{G}_{\mathrm{ML}}^{s}(V)=\underset{h \nearrow_{\infty}}{\lim _{\mathrm{ML}}} \mathrm{G}_{\mathrm{ML}}^{s, h}(V)
$$

são Hausdorff e Montel com a topologia de limite indutivo. Em particular, seqüências limitadas em algum $\mathrm{G}_{\mathrm{ML}}^{s, h}(V)$ admitem subseqüências convergentes em $\mathrm{G}_{\mathrm{ML}}^{s, h+}(V)$ para todo $h_{+}>h$ e, conseqüentemente, em $\mathrm{G}_{\mathrm{ML}}^{s}(V)$.

Observação 5 Se $\Phi=0$ ou, mais geralmente se $\Phi \in \mathcal{C}^{w}$ as classes de funções Gevrey usuais $\mathrm{G}^{s, h}$ e as classes $\mathrm{G}_{\mathrm{ML}}^{s, h}$ coincidem.

\subsection{O conceito de hipoelipticidade Gevrey}

O objetivo desta seção é estudar a regularidade Gevrey das soluções de

$$
\mathrm{L} u=f .
$$

Para ser mais preciso vamos estudar a hipoelipticidade $G^{s}$ e a hipoelipticidade $G_{M L}^{s}$ de $L$ que definimos a seguir.

Definição 2.4 Seja $\mathcal{V}$ uma estrutura localmente integrável analítica sobre uma variedade analítica real $\Omega$. Dizemos que $\mathcal{V}$ é $G^{s}$-hipoelíptica sobre $W \subset$ $\Omega$ aberto arbitrário se dados $W_{0} \subset W$ aberto e $u \in \mathcal{D}^{\prime}\left(W_{0}\right)$ então

$$
d_{0}^{\prime} u \in \wedge^{1} G^{s}\left(W_{0}\right) \Rightarrow u \in G^{s}\left(W_{0}\right)
$$


Retornemos á notação da seção 2.2 .

Definição 2.5 Dizemos que $\mathrm{L}$ é $\mathrm{G}_{\mathrm{ML}}^{s}$-hipoelíptico em 0 se dada $u \in \mathcal{D}^{\prime}$ em uma vizinhança aberta da origem a condição $\mathrm{L} u \in \wedge^{1} \mathrm{G}_{\mathrm{ML}}^{s}$ numa vizinhança da origem implica $u \in \mathrm{G}_{\mathrm{ML}}^{s}$ numa vizinhança da origem.

\subsection{O teorema principal}

O resultado central é o seguinte

Teorema 2 Seja $\mathrm{L}=d_{t}-i d_{t} \Phi \wedge \frac{\partial}{\partial x}$, onde $\Phi: \Theta \subset \mathbf{R}^{n} \rightarrow \mathbf{R}, \Phi(0)=0$, é uma função $\mathcal{C}^{\infty}$ sem mínimo nem máximo local satisfazendo a seguinte propriedade:

- Existem $M>0$ e $0 \leq \theta<1$ tais que

$$
|\nabla \Phi| \geq M|\Phi|^{\theta} \quad \text { em } \quad \Theta .
$$

Então $\mathrm{L}$ é $\mathrm{G}_{\mathrm{ML}}^{s}$-hipoelíptico em 0 para todo $s \geq 1$.

Observe que aparentemente a condição (2.8) parece restritiva demais. Entretanto é possível provar (cf. [L], p.92) que toda função analítica real satisfaz uma desigualdade do tipo (2.8):

Proposição 2.2 [L] Seja $\varphi$ uma função analítica definida em uma vizinhança de um ponto $a \in \mathbf{R}^{n}$. Então existe $\left.\theta \in\right] 0,1[$ tal que

$$
|\nabla \varphi(t)| \geq|\varphi(t)-\varphi(a)|^{\theta}
$$

em uma vizinhança de a.

Corolário 2.1 Seja $\mathcal{V}$ uma estrutura hipocomplexa tubular analítica sobre $\Omega$ com $m=1$. Então $\mathcal{V}$ é $G^{s}$-hipoeliptica sobre $W \subset \Omega$ aberto arbitrário para todo $s \geq 1$. 


\subsubsection{Demonstração do teorema principal: Preliminares}

O lema a seguir nos dá o controle do crescimento ( e decrescimento) de $\varphi$ ao longo do fluxo do campo gradiente.

Usaremos as seguintes desigualdades, cuja verificações são imediatas:

Para cada $\sigma \geq 1$ existem constantes $C_{\sigma}, C_{\sigma}^{\prime}>0$ tais que

$$
\begin{aligned}
(x-y)^{\sigma} & \leq C_{\sigma}\left(x^{\sigma}-y^{\sigma}\right), \quad \forall x \geq y \geq 0 \\
x^{\sigma}+y^{\sigma} & \geq C_{\sigma}^{\prime}(x+y)^{\sigma} \quad \forall x, y \geq 0
\end{aligned}
$$

Lema 8 Sejam $\Theta \subseteq \mathbf{R}^{n}$ um aberto e $\varphi \in \mathcal{C}^{\infty}(\bar{\Theta})$ uma função real que satisfaz a condição (2.8). Defina $\Sigma=\{t \in \Theta \mid \nabla \varphi(t)=0\}$ e considere, para cada $t \in \Theta \backslash \Sigma, \gamma_{t}(\tau)$ solução de

$$
\left\{\begin{array}{lll}
\dot{\gamma}_{t}(\tau) & = & -\frac{\nabla \varphi}{\mid \nabla \varphi}\left(\gamma_{t}(\tau)\right) \\
\gamma_{t}(0) & = & t
\end{array}\right.
$$

definida em $[0, \delta(t)[$. Então existem constantes $C>0$ e $\sigma \geq 1$ tais que

$$
\varphi(t)-\varphi\left(\gamma_{t}(\tau)\right) \geq C \tau^{\sigma}, \quad \forall \tau \in[0, \delta(t)[.
$$

Observação 6 A desigualdade acima implica que $\delta(t)$ é uniformemente limitada em $\Theta$.

\section{Demonstração :}

$\triangleright \quad$ Seja $h_{t}=\varphi \circ \gamma_{t}$. Assim

$$
\begin{aligned}
\dot{h}_{t}(\tau) & =\nabla \varphi\left(\gamma_{t}(\tau)\right) \cdot \dot{\gamma}_{t}(\tau) \\
& =-\left|\nabla \varphi\left(\gamma_{t}(\tau)\right)\right| \\
& \leq-M\left|\varphi\left(\gamma_{t}(\tau)\right)\right|^{\theta} \\
& =-M\left|h_{t}(\tau)\right|^{\theta}, \quad \forall \tau \in[0, \delta(t)[.
\end{aligned}
$$

Temos dois casos a considerar: 
$\left(1^{0}\right) h_{t}(0)=\varphi(t) \leq 0$.

Como $\varphi$ é estritamente decrescente ao longo de $\gamma_{t}$ segue que $h_{t}(\tau) \leq 0, \forall \tau \in[0, \delta(t)[$.

Assim

$$
\dot{h}_{t}(\tau) \leq-M\left(-h_{t}(\tau)\right)^{\theta}
$$

ou seja

$$
\frac{-\dot{h}_{t}(\tau)}{\left(-h_{t}(\tau)\right)^{\theta}} \geq M, \quad \forall \tau \in(0, \delta(t)[.
$$

Note que não há possibilidade de que $h_{t}(\tau)=0$ a menos que $\tau=0$ e portanto o quociente acima está bem definido.

Integrando de 0 a $\tau$ obtemos

$$
\frac{1}{1-\theta}\left[\left(-h_{t}(\tau)\right)^{1-\theta}-\left(-h_{t}(0)\right)^{1-\theta}\right] \geq M \tau
$$

donde, usando

$$
\sigma=\frac{1}{1-\theta} \geq 1
$$

obtemos por (2.9)

$$
\begin{aligned}
C_{\sigma}\left[-h_{t}(\tau)+h_{t}(0)\right] & \geq\left[\left(-h_{t}(\tau)\right)^{1-\theta}-\left(-h_{t}(0)\right)^{1-\theta}\right]^{\sigma} \\
& \geq[M(1-\theta) \tau]^{\sigma}, \quad \forall \tau \in[0, \delta(t)[.
\end{aligned}
$$

$\left(2^{0}\right) \quad h_{t}(0)>0$.

Seja $\alpha \in[0, \delta(t)[$ tal que

$$
\begin{aligned}
& h_{t}(\tau)>0, \quad \forall \tau \in[0, \alpha[ \\
& h_{t}(\tau) \leq 0, \quad \forall \tau \in[\alpha, \delta(t)[.
\end{aligned}
$$

Observe que $\alpha$ está bem definido porque $\varphi$ é decrescente ao longo de $\gamma_{t}$ e que no caso $\alpha=\delta(t)$ temos simplesmente que $h_{t}(\tau)>0$, 
$\forall \tau \in[0, \delta(t)[$.

Se $\tau \in[0, \alpha[$ então

$$
\begin{aligned}
\int_{0}^{\tau} \frac{\dot{h}_{t}(s)}{\left|h_{t}(s)\right|^{\theta}} d s & =\int_{0}^{\tau} \frac{\dot{h}_{t}(s)}{h_{t}(s)^{\theta}} d s \\
& \leq-M \tau .
\end{aligned}
$$

e portanto usando que $\sigma=1 /(1-\theta)$ obtemos por (2.9)

$$
C_{\sigma}\left[h_{t}(0)-h_{t}(\tau)\right] \geq[(1-\theta) M \tau]^{\sigma}, \quad \forall \tau \in[0, \alpha[.
$$

Observe que, por continuidade, fazendo $\tau \nearrow \alpha$ temos

$$
h_{t}(0)-h_{t}(\alpha) \geq C \alpha^{\sigma} \text {. }
$$

Agora se $\tau \in[\alpha, \delta(t)[$ temos

$$
\begin{aligned}
h_{t}(0)-h_{t}(\tau) & =h_{t}(0)-h_{t}(\alpha)+h_{t}(\alpha)-h_{t}(\tau) \\
& \geq C \alpha^{\sigma}+h_{\gamma_{t}(\alpha)}(0)-h_{\gamma_{t}(\alpha)}(\tau-\alpha) \quad \text { (o sistema é autônomo) } \\
& \geq C\left[\alpha^{\sigma}+(\tau-\alpha)^{\sigma}\right] \\
& \geq C^{\prime} \tau^{\sigma} \quad \text { por }(2.10) .
\end{aligned}
$$

Observação 7 (i) Com as hipóteses do Lema existe o limite

$$
\lim _{\tau-, \delta(t)^{-}} \gamma_{t}(\tau)
$$

para cada $t \in \Theta \backslash \Sigma$.

De fato, seja $\left(\tau_{j}\right) \subset\left[0, \delta(t)\left[\right.\right.$ uma seqüência tal que $\tau_{j} \rightarrow \delta(t)<+\infty$. Usando que $\left|\dot{\gamma}_{t}(\tau)\right|=1, \forall \tau \in[0, \delta(t)[$ temos

$$
\left|\gamma_{t}\left(\tau_{j}\right)-\gamma_{t}\left(\tau_{k}\right)\right| \leq\left|\tau_{j}-\tau_{k}\right|
$$

o que implica que $\left(\gamma_{t}\left(\tau_{j}\right)\right)$ é uma seqüencia de Cauchy em $\Theta$ e portanto convergente para um ponto $l(t) \in \bar{\Theta}$. 
(ii) Note que $l(t)$ não depende da seqüência $\left(\tau_{j}\right)$ : se $\left(\tau_{j}^{\prime}\right) \subset[0, \delta(t)[$ com $\tau_{j}^{\prime} \rightarrow \delta(t)$ então

$$
\left|\gamma_{t}\left(\tau_{j}\right)-\gamma_{t}\left(\tau_{j}^{\prime}\right)\right| \leq\left|\tau_{j}-\tau_{j}^{\prime}\right|
$$

implica que

$$
\lim _{j \rightarrow \infty} \gamma_{t}\left(\tau_{j}\right)=\lim _{j \rightarrow \infty} \gamma_{t}\left(\tau_{j}^{\prime}\right)=l(t)
$$

uma vez que $\delta(t)$ é finito.

(iii) Note $l(t) \in \partial \Theta \cup \Sigma$ porque caso contrário $\delta(t)$ não seria o extremo direito do intervalo maximal de $\gamma_{t}$.

Usando novamente que $\varphi$ é estritamente decrescente ao longo de $\gamma_{t}$, se $\varphi(t)<0$ então $l(t) \in \partial \Theta$ porque $\varphi(t)=0, \forall t \in \Sigma$ Além disso, se $l(t) \in \Sigma$ então $\varphi(t)>0$.

\subsubsection{Demonstração do teorema principal: Conclusão.}

\section{Demonstração :}

$\triangleright \quad$ No caso $s=1$ o resultado segue do teorema 2.1 de [BT1]. Vamos supor $s>1$. Seja $U=]-a, a\left[\times \Theta\right.$ uma vizinhança da origem e $u \in \mathcal{D}^{\prime}(U)$ tal que $\mathrm{L} u \in \wedge^{1} \mathrm{G}_{\mathrm{ML}}^{s, h}(U)$. Pelo corolário 4.2.1 de $[\mathrm{A}]$ segue-se que $u \in$ $\mathcal{C}^{\infty}(U)$.

Nosso objetivo é mostrar que $u$ satisfaz (2.7). Podemos assumir que $\alpha_{2}=0$ pois, caso contrário fazemos $\alpha_{2}=\alpha_{2}^{\prime}+e_{j}$ assim

$$
\begin{aligned}
\sup _{\bar{U}}\left|\mathrm{M}^{\alpha_{1}} L^{\alpha_{2}} u\right| & =\sup _{\bar{U}}\left|\mathrm{M}^{\alpha_{1}} \mathrm{~L}^{\alpha_{2}^{\prime}} f_{j}\right| \\
& \leq \text { const } \cdot h^{\left|\alpha_{1}\right|+\left|\alpha_{2}^{\prime}\right|}\left(\left|\alpha_{1}\right|+\left|\alpha_{2}^{\prime}\right|\right) !^{s} \\
& \leq \text { const } \cdot h^{\left|\alpha_{1}\right|+\left|\alpha_{2}\right|}\left(\left|\alpha_{1}\right|+\left|\alpha_{2}\right|\right) !^{s}, \forall\left(\alpha_{1}, \alpha_{2}\right) \in \mathrm{Z}_{+}^{1+n} .
\end{aligned}
$$


Seja $g \in G^{s}(\mathbf{R}) \cap \mathcal{C}_{c}^{\infty}(]-a, a[) \operatorname{com} g \equiv 1$ para $|x| \leq a^{\prime}, a^{\prime}<a$. Para $(x, t) \in \mathbf{R} \times \Theta^{\prime}, \Theta^{\prime} \subset \subset \Theta$, e $\epsilon>0$ considere as integrais

$$
\begin{gathered}
\mathcal{K}_{\epsilon}^{+}(x, t)=\frac{1}{2 \pi} \int_{0}^{\infty} \int_{-\infty}^{\infty} \int_{\gamma} e^{i\left[Z(x, t)-Z\left(x^{\prime}, t^{\prime}\right)\right] \xi-\epsilon \xi^{2}} g\left(x^{\prime}\right) \mathrm{L}\left[u\left(x^{\prime}, t^{\prime}\right)\right] d t^{\prime} d x^{\prime} d \xi \\
\mathcal{K}_{\epsilon}^{-}(x, t)=\frac{1}{2 \pi} \int_{-\infty}^{0} \int_{-\infty}^{\infty} \int_{\gamma} e^{i\left[Z(x, t)-Z\left(x^{\prime}, t^{\prime}\right)\right] \xi-\epsilon \xi^{2}} g\left(x^{\prime}\right) \mathrm{L}\left[u\left(x^{\prime}, t^{\prime}\right)\right] d t^{\prime} d x^{\prime} d \xi
\end{gathered}
$$

onde $\gamma$ é uma curva Lipschitz em $\Theta$ com ponto inicial $t$ e ponto final $t^{*}$. Usando que $g \mathrm{~L} u=\mathrm{L}[g u]-u \mathrm{~L}[g]$ temos que

$$
\begin{aligned}
\mathcal{K}_{\epsilon}^{+}(x, t) & =\frac{1}{2 \pi} \int_{0}^{\infty} \int_{-\infty}^{\infty} \int_{\gamma} e^{i\left[Z(x, t)-Z\left(x^{\prime}, t^{\prime}\right)\right] \xi-\epsilon \xi^{2}} L\left[g\left(x^{\prime}\right) u\left(x^{\prime}, t^{\prime}\right)\right] d t^{\prime} d x^{\prime} d \xi \\
& -\frac{1}{2 \pi} \int_{0}^{\infty} \int_{-\infty}^{\infty} \int_{\gamma} e^{i\left[Z(x, t)-Z\left(x^{\prime}, t^{\prime}\right)\right] \xi-\epsilon \xi^{2}} L\left[g\left(x^{\prime}\right)\right] u\left(x^{\prime}, t^{\prime}\right) d t^{\prime} d x^{\prime} d \xi
\end{aligned}
$$

Integrando por partes e usando (2.6) obtemos

$$
\begin{aligned}
\mathcal{K}_{\epsilon}^{+}(x, t)=\frac{1}{2 \pi} \int_{0}^{\infty} \int_{-\infty}^{\infty} e^{i\left(x-x^{\prime}\right) \xi-\left(\Phi(t)-\Phi\left(t^{*}\right)\right) \xi-\epsilon \xi^{2}} g\left(x^{\prime}\right) u\left(x^{\prime}, t^{*}\right) d x^{\prime} d \xi \\
-\frac{1}{2 \pi} \int_{0}^{\infty} \int_{-\infty}^{\infty} e^{i \xi\left(x-x^{\prime}\right)-\epsilon \xi^{2}} g\left(x^{\prime}\right) u\left(x^{\prime}, t\right) d x^{\prime} d \xi- \\
\quad-\frac{1}{2 \pi} \int_{0}^{\infty} \int_{\left|x^{\prime}\right| \geq a^{\prime}} \int_{\gamma} e^{i\left[Z(x, t)-Z\left(x^{\prime}, t^{\prime}\right)\right] \xi-\epsilon \xi^{2}} \mathrm{~L}\left[g\left(x^{\prime}\right)\right] u\left(x^{\prime}, t^{\prime}\right) d t^{\prime} d x^{\prime} d \xi \\
=\mathcal{H}_{\epsilon}{ }^{+}(x, t)-u_{\epsilon}{ }^{+}(x, t)-\mathcal{R}_{\epsilon}{ }^{+}(x, t) .
\end{aligned}
$$

De forma análoga,

$$
\mathcal{K}_{\epsilon}^{-}(x, t)=\mathcal{H}_{\epsilon}^{-}(x, t)-u_{\epsilon}{ }^{-}(x, t)-\mathcal{R}_{\epsilon}{ }^{-}(x, t) .
$$

Observe que $u_{\epsilon}{ }^{+}$e $u_{\epsilon}{ }^{-}$convergem em $\mathcal{C}^{\infty}(U)$ respectivamente para funções $u^{+}$e $u^{-}$e que $u^{+}+u^{-}=u$. Além disso, existem $U_{0} \subset \subset U$ e $h_{0}>0$ tais que

$$
\sup _{\overline{U_{0}, \epsilon>0}}\left|\partial_{x}^{l} \mathcal{R}_{\epsilon}^{ \pm}(x, t)\right| \leq \text { Const } \cdot h_{0}^{l} l !^{s} \quad \forall l \in \mathbf{Z}_{+} .
$$


De fato, $\mathcal{R}_{\epsilon}^{+}(x, t)=\frac{1}{2 \pi} \int_{0}^{\infty} \int_{\left|x^{\prime}\right|>a^{\prime}} \int_{\gamma} e^{i\left[Z(x, t)-Z\left(x^{\prime}, t^{\prime}\right)\right] \xi-\epsilon \xi^{2}} L\left[g\left(x^{\prime}\right)\right] u\left(x^{\prime}, t^{\prime}\right) d t^{\prime} d x^{\prime} d \xi$. Para $|x|<a^{\prime} / 2, \xi \in \mathbf{R}_{+}$e para cada $\epsilon>0$ consideremos a seguinte função inteira em $\mathbb{C}$ :

$F_{\epsilon}(x+i y, t)=\int_{0}^{\infty} \int_{\left|x^{\prime}\right|>a^{\prime}} \int_{\gamma} e^{i\left[x+i y+i \Phi(t)-x^{\prime}-i \Phi\left(t^{\prime}\right)\right] \xi-\epsilon \xi^{2}} L\left[g\left(x^{\prime}\right)\right] u\left(x^{\prime}, t^{\prime}\right) d t^{\prime} d x^{\prime} d \xi$ com $|y|<a^{\prime} / 8$. Considerando a deformação do dominío de integração em $\xi$ para $\xi \mapsto \zeta=\xi\left(1+i \frac{x-x^{\prime}}{2\left|x-x^{\prime}\right|}\right)$ obtemos que a parte real do expoente é igual a

$$
Q \doteq-\frac{\left|x-x^{\prime}\right|}{2} \xi+\left(\Phi\left(t^{\prime}\right)-\Phi(t)\right) \xi-3 / 4 \xi^{2} \epsilon-y \xi
$$

Como $\left|x-x^{\prime}\right| \geq a^{\prime} / 2$ e $\Phi$ é decrescente ao longo de $\gamma$ então

$$
\begin{aligned}
Q & \leq-a^{\prime} / 4 \xi-3 / 4 \xi^{2} \epsilon-y \xi \\
& =-\left(a^{\prime} / 4+y\right) \xi-3 / 4 \xi^{2} \epsilon .
\end{aligned}
$$

Portanto para $|y|<a^{\prime} / 8$ a integral

$$
\begin{aligned}
\left|F_{\epsilon}(x+i y, t)\right| & \leq \text { Const. } \int_{0}^{\infty} e^{-\left(a^{\prime} / 4+y\right) \xi-3 / 4 \xi^{2} \epsilon} d \xi \\
& \leq \text { Const. }
\end{aligned}
$$

Assim pelas estimativas de Cauchy existem $U_{0} \subset \subset U$ e $h_{0}>0$ tais que

$$
\sup _{\overline{U_{0}, \epsilon>0}}\left|\partial_{x}^{l} \mathcal{R}_{\epsilon}^{+}(x, t)\right| \leq \text { Const } \cdot h_{0}^{l} l !^{s} \quad \forall l \in \mathrm{Z}_{+} .
$$

De forma análoga se obtém a estimativa (2.12) para $\mathcal{R}_{\varepsilon}^{-}$.

Então para demonstrar o Teorema é suficiente mostrar existe $h>0$ tais que

$$
\sup _{\overline{U_{0}, \epsilon>0}}\left|\mathrm{M}^{l} \mathcal{K}_{\epsilon}^{ \pm}(x, t)\right| \leq \text { Const } \cdot h^{l} l !^{s}
$$




$$
\sup _{\overline{U_{0}}, \epsilon>0}\left|\mathrm{M}^{l} \mathcal{H}_{\epsilon}^{ \pm}(x, t)\right| \leq \text { Const } \cdot h^{l} l !^{s},
$$

De fato, se $h^{*}=\max \left\{h_{0}, h\right\}$

$$
\begin{aligned}
\sup _{\overline{U_{0}}, \epsilon>0}\left|\mathrm{M}^{l}\left(u_{\epsilon}^{+}+u_{\epsilon}^{-}\right)(x, t)\right| \leq & \sup _{\overline{U_{0}}, \epsilon>0}\left|\mathrm{M}^{l} \mathcal{K}_{\epsilon}^{+}(x, t)\right|+\sup _{\overline{U_{0}}, \epsilon>0}\left|\mathrm{M}^{l} \mathcal{K}_{\epsilon}^{-}(x, t)\right|+ \\
& +\sup _{\overline{U_{0}}, \epsilon>0}\left|\mathrm{M}^{l} \mathcal{H}_{\epsilon}^{+}(x, t)\right|+\sup _{\overline{U_{0}}, \epsilon>0}\left|\mathrm{M}^{l} \mathcal{H}_{\epsilon}^{-}(x, t)\right|+ \\
& +\sup _{\overline{U_{0}}, \epsilon>0}\left|\mathrm{M}^{l} \mathcal{R}_{\epsilon}^{+}(x, t)\right|+\sup _{\overline{U_{0}}, \epsilon>0}\left|\mathrm{M}^{l} \mathcal{R}_{\epsilon}^{-}(x, t)\right| \\
\leq & \text { Const } \cdot h^{* l} l !^{s}, \quad \forall l \in \mathrm{Z}_{+},
\end{aligned}
$$

concluindo assim que

$$
u \in \mathrm{G}_{\mathrm{ML}}^{s, h^{*}}\left(U_{0}\right) .
$$

Para $t \in \Theta^{\prime}$ vamos tratar dois casos:

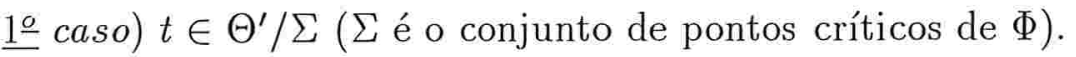

Pelo lema 8 existe uma curva $\gamma_{t}$ começando em $t$ e finalizando em $l(t)$. Primeiro vamos considerar $l(t) \in \partial \Theta$.

Defina

$$
d^{\prime}=\frac{\operatorname{dist}\left(\Theta^{\prime}, \partial \Theta\right)}{2}>0
$$

Se $t \in \Theta^{\prime} / \Sigma$ então, pelo lema 8 ,

$$
\begin{aligned}
\Phi(t)-\Phi(l(t)) & \geq C \delta(t)^{\sigma} \\
& \geq C|l(t)-t|^{\sigma} \\
& \geq C d^{\sigma \sigma}
\end{aligned}
$$

pois $\gamma_{t}$ é uma curva parametrizada por comprimento de arco. Vamos definir

$$
d=C d^{\prime \sigma}>0
$$

Observando que

$$
\mathrm{M}^{l}\left[e^{i \xi\left[Z(x, t)-Z\left(x^{\prime}, t^{\prime}\right)\right]-\epsilon \xi^{2}}\right]=(-1)^{l} \mathrm{M}^{\prime l}\left[e^{i \xi\left[Z(x, t)-Z\left(x^{\prime}, t^{\prime}\right)\right]-\epsilon \xi^{2}}\right],
$$


onde $\mathrm{M}^{\prime}$ é o operador $\mathrm{M}$ nas variável $\left(x^{\prime}, t^{\prime}\right)$, e integrando por partes, segue que

$$
\begin{aligned}
& \mathrm{M}^{l} \mathcal{K}_{\epsilon}^{+}(x, t)=\mathrm{M}^{l}\left[\frac{1}{2 \pi} \int_{0}^{\infty} \int_{-\infty}^{\infty} \int_{\gamma_{t}} e^{i \xi\left[Z(x, t)-Z\left(x^{\prime}, t^{\prime}\right)\right]-\epsilon \xi^{2}}\right. \\
& \left.\times g\left(x^{\prime}\right) \mathrm{L} u\left(x^{\prime}, t^{\prime}\right) d x^{\prime} d t^{\prime} d \xi\right] \\
= & \frac{1}{2 \pi} \int_{0}^{\infty} \int_{-a}^{a} \int_{\gamma_{t}} \mathrm{M}^{l}\left[e^{i\left[Z(x, t)-Z\left(x^{\prime}, t^{\prime}\right)\right] \xi-\epsilon \xi^{2}}\right] g\left(x^{\prime}\right) \mathrm{L} u\left(x^{\prime}, t^{\prime}\right) d t^{\prime} d x^{\prime} d \xi \\
= & \frac{(-1)^{l}}{2 \pi} \int_{0}^{\infty} \int_{-a}^{a} \int_{\gamma_{t}} \mathrm{M}^{\prime l}\left[e^{i\left[Z(x, t)-Z\left(x^{\prime}, t^{\prime}\right)\right] \xi-\epsilon \xi^{2}}\right] g\left(x^{\prime}\right) \mathrm{L} u\left(x^{\prime}, t^{\prime}\right) d t^{\prime} d x^{\prime} d \xi \\
= & \frac{1}{2 \pi} \int_{0}^{\infty} \int_{-a}^{a} \int_{\gamma_{t}} e^{i\left[Z(x, t)-Z\left(x^{\prime}, t^{\prime}\right)\right] \xi-\epsilon \xi^{2}} \mathrm{M}^{\prime l}\left[g\left(x^{\prime}\right) L u\left(x^{\prime}, t^{\prime}\right)\right] d t^{\prime} d x^{\prime} d \xi .
\end{aligned}
$$

Assim

$$
\begin{aligned}
\left|\mathrm{M}^{l} \mathcal{K}_{\epsilon}^{+}(x, t)\right| & =\mid \frac{1}{2 \pi} \int_{0}^{\infty} \int_{-a}^{a} \int_{\gamma_{t}}(1+\xi)^{-2} e^{i\left[Z(x, t)-Z\left(x^{\prime}, t^{\prime}\right)\right] \xi-\epsilon \xi^{2}} \times \\
& \times\left[1-i \mathrm{M}^{\prime}\right]^{2} \mathrm{M}^{\prime l}\left[g\left(x^{\prime}\right) \mathrm{L} u\left(x^{\prime}, t^{\prime}\right)\right] d t^{\prime} d x^{\prime} d \xi \mid \\
\leq & \frac{1}{2 \pi} \int_{0}^{\infty} \int_{-a}^{a} \int_{\gamma_{t}}(1+\xi)^{-2} e^{\left[\Phi\left(t^{\prime}\right)-\Phi(t)\right] \xi-\epsilon \xi^{2}} \times \\
& \times\left|\left[1-i \mathrm{M}^{\prime}\right]^{2} \mathrm{M}^{\prime l}\left[g\left(x^{\prime}\right) \mathrm{L} u\left(x^{\prime}, t^{\prime}\right)\right]\right| d t^{\prime} d x^{\prime} d \xi \\
\leq & \frac{1}{2 \pi} \int_{0}^{\infty} \int_{-a}^{a} \int_{0}^{\delta(t)}(1+\xi)^{-2} e^{-C \tau^{\sigma} \xi-\epsilon \xi^{2} \times} \\
\leq & \quad\left|\left[1-i \mathrm{M}^{\prime}\right]^{2} \mathrm{M}^{\prime l}\left[g\left(x^{\prime}\right) \mathrm{L} u\left(x^{\prime}, \gamma_{t}(\tau)\right)\right]\right| d \tau d x^{\prime} d \xi \\
\leq & \text { const } \cdot \sup _{\bar{U}}\left|[1-i \mathrm{M}]^{2} \mathrm{M}^{l}[g(x) \mathrm{L} u(x, t)]\right| \int_{0}^{\infty}(1+\xi)^{-2} d \xi \\
\leq & \text { Const } \cdot h^{(l+2)}((l+2) !)^{s} \leq \text { Const } \cdot h^{2}(l+2)^{s}(l+1)^{s} h^{l}(l !)^{s} \\
\leq & \text { Const } \cdot h^{2}\left(8 \times 4^{l}\right)^{s} h^{l}(l !)^{s} \\
\leq & \text { Const } \cdot h^{2} 8^{s}\left(4^{s} h\right)^{l}(l !)^{s} .
\end{aligned}
$$


Agora estimamos $\left|\mathrm{M}^{l} \mathcal{H}_{\epsilon}^{+}\right|$.

$$
\begin{aligned}
& \mathrm{M}^{l} \mathcal{H}_{\epsilon}^{+}(x, t)=\partial_{x}^{l} \mathcal{H}_{\epsilon}^{+}(x, t) \\
& =\partial_{x}^{l}\left[\frac{1}{2 \pi} \int_{0}^{\infty} \int_{-a}^{a} e^{i\left(x-x^{\prime}\right) \xi-(\Phi(t)-\Phi(l(t))) \xi-\epsilon \xi^{2}} g\left(x^{\prime}\right) u\left(x^{\prime}, l(t)\right) d x^{\prime} d \xi\right] \\
& =\frac{1}{2 \pi} \int_{0}^{\infty} \int_{-a}^{a} \partial_{x}^{l}\left[e^{i\left(x-x^{\prime}\right) \xi-(\Phi(t)-\Phi(l(t))) \xi-\epsilon \xi^{2}}\right] g\left(x^{\prime}\right) u\left(x^{\prime}, l(t)\right) d x^{\prime} d \xi .
\end{aligned}
$$

Assim obtemos

$$
\begin{aligned}
\left|\mathrm{M}^{l} \mathcal{H}_{\epsilon}^{+}(x, t)\right| & \leq\left|\frac{1}{2 \pi} \int_{0}^{\infty} \int_{-a}^{a}(i)^{l} \xi^{l} e^{i\left(x-x^{\prime}\right) \xi-d \xi-\epsilon \xi^{2}} g\left(x^{\prime}\right) u\left(x^{\prime}, l(t)\right) d x^{\prime} d \xi\right| \\
& \leq \frac{1}{2 \pi} \int_{0}^{\infty} \int_{-a}^{a} \xi^{l} e^{-\left(d \xi+\epsilon \xi^{2}\right)}\left|g\left(x^{\prime}\right) u\left(x^{\prime}, l(t)\right)\right| d x^{\prime} d \xi \\
& =\frac{1}{2 \pi} \int_{0}^{\infty} \int_{-a}^{a}\left(\xi^{l} e^{-\frac{d}{2} \xi}\right) e^{-\frac{d}{2} \xi-\epsilon \xi^{2}}\left|g\left(x^{\prime}\right) u\left(x^{\prime}, l(t)\right)\right| d x^{\prime} d \xi
\end{aligned}
$$

Como

$$
\xi^{l} e^{-\frac{d}{2} \xi} \leq\left(\frac{2 l}{d}\right)^{l} e^{-\frac{d}{2}\left(\frac{2 l}{d}\right)}
$$

obtemos que

$$
\begin{aligned}
\left|\partial_{x}^{l} \mathcal{H}_{\epsilon}^{+}(x, t)\right| & =\frac{1}{2 \pi} \int_{0}^{\infty} \int_{-a}^{a}\left(\frac{2 l}{d}\right)^{l} \xi^{l} e^{-\frac{d}{2}\left(\frac{2 l}{d}\right)} e^{\left.-\frac{d}{2} \xi-\epsilon \xi^{2}\right)}\left|g\left(x^{\prime}\right) u\left(x^{\prime}, l(t)\right)\right| d x^{\prime} d \xi . \\
& =\left(\frac{2 l}{d}\right)^{l}(2 \pi)^{-1} e^{-l} \int_{0}^{\infty} \int_{-a}^{a} e^{-\frac{d}{2} \xi-\epsilon \xi^{2}}\left|g\left(x^{\prime}\right) u\left(x^{\prime}, l(t)\right)\right| d x^{\prime} d \xi \\
& \leq\left(\frac{2 l}{d}\right)^{l}(2 \pi)^{-1} e^{-l} 2 a \sup _{\bar{U}}\left|g\left(x^{\prime}\right) u\left(x^{\prime}, l(t)\right)\right| \int_{0}^{\infty} e^{-\frac{d}{2} \xi-\epsilon \xi^{2}} d \xi \\
& \leq\left(\frac{2}{d}\right)^{l}\left(l ! e^{l}\right)(2 \pi)^{-1} e^{-l} 2 a \cdot \text { Const (fórmula de Stirling) } \\
& \leq \text { Const } \cdot\left(\frac{2}{d}\right)^{l} l !
\end{aligned}
$$

Quando $l(t) \in \Sigma$ ( e portanto $\Phi(t)>0$ e $\Phi(l(t))=0$ ), escolhemos $t_{0}$ arbitrariamente próximo de $l(t)$ satisfazendo as seguintes condições:

- $\Phi\left(t_{0}\right)<0$ (lembre que $\Phi$ não tem mínimo local). 
- $t_{0} \in V$, onde $V$ é uma vizinhança aberta convexa de $l(t)$ na qual $\Phi(s)<$ $\Phi(t), \forall s \in V$.

- $\operatorname{Se} \operatorname{dist}(l(t), \partial \Theta)>d^{\prime}$ então supomos que $\operatorname{dist}\left(t_{0}, \partial \Theta\right)>\mathrm{d}^{\prime}$.

Note que, com a primeira condição acima, $l\left(t_{0}\right) \in \partial \Theta$. Aqui escolhemos $\gamma=\gamma_{t} \cup\left[l(t), t_{0}\right] \cup \gamma_{t_{0}}$, onde $\gamma_{t}$ e $\gamma_{t_{0}}$ são como no lema 8 e $\left[l(t), t_{0}\right]$ é o segmento que une $l(t)$ com $t_{0}$. Então

$$
\begin{aligned}
\mathrm{M}^{l} \mathcal{K}_{\epsilon}^{+}(x, t) & =\frac{1}{2 \pi} \int_{0}^{\infty} \int_{-a}^{a} \int_{\gamma} e^{i\left[Z(x, t)-Z\left(x^{\prime}, t^{\prime}\right)\right] \xi-\epsilon \xi^{2}} \mathrm{M}^{\prime l}\left[g\left(x^{\prime}\right) \mathrm{L} u\left(x^{\prime}, t^{\prime}\right)\right] d x^{\prime} d t^{\prime} d \xi \\
& =\frac{1}{2 \pi} \int_{0}^{\infty} \int_{-a}^{a} \int_{\gamma_{t}} e^{i\left[Z(x, t)-Z\left(x^{\prime}, t^{\prime}\right)\right] \xi-\epsilon \xi^{2}} \mathrm{M}^{\prime l}\left[g\left(x^{\prime}\right) \mathrm{L} u\left(x^{\prime}, t^{\prime}\right)\right] d x^{\prime} d t^{\prime} d \xi \\
& +\frac{1}{2 \pi} \int_{0}^{\infty} \int_{-a}^{a} \int_{\left[l(t), t_{0}\right]} e^{i\left[Z(x, t)-Z\left(x^{\prime}, t^{\prime}\right)\right]-\epsilon \xi^{2}} \mathrm{M}^{\prime l}\left[g\left(x^{\prime}\right) \mathrm{L} u\left(x^{\prime}, t^{\prime}\right)\right] d x^{\prime} d t^{\prime} d \xi \\
& +\frac{1}{2 \pi} \int_{0}^{\infty} \int_{-a}^{a} \int_{\gamma_{t_{0}}} e^{i \xi\left[Z(x, t)-Z\left(x^{\prime}, t^{\prime}\right)\right] \xi-\epsilon \xi^{2} \mathrm{M}^{\prime l}}\left[g\left(x^{\prime}\right) \mathrm{L} u\left(x^{\prime}, t^{\prime}\right)\right] d x^{\prime} d t^{\prime} d \xi .
\end{aligned}
$$

\section{Portanto}

$$
\begin{aligned}
& \left|\mathrm{M}^{l} \mathcal{K}_{\epsilon}^{+}(x, t)\right| \leq \frac{1}{2 \pi}\left|\int_{0}^{\infty} \int_{-a}^{a} \int_{\gamma_{t}} e^{i\left[Z(x, t)-Z\left(x^{\prime}, t^{\prime}\right)\right] \xi-\epsilon \xi^{2}} \mathrm{M}^{\prime l}\left[g\left(x^{\prime}\right) \mathrm{L} u\left(x^{\prime}, t^{\prime}\right)\right] d t^{\prime} d x^{\prime} d \xi\right|+ \\
& +\frac{1}{2 \pi}\left|\int_{0}^{\infty} \int_{-a}^{a} \int_{\left[l(t), t_{0}\right]} e^{i\left[Z(x, t)-Z\left(x^{\prime}, t^{\prime}\right)\right] \xi-\epsilon \xi^{2}} \mathrm{M}^{\prime l}\left[g\left(x^{\prime}\right) \mathrm{L} u\left(x^{\prime}, t^{\prime}\right)\right] d t^{\prime} d x^{\prime} d \xi\right| \\
& +\frac{1}{2 \pi}\left|\int_{0}^{\infty} \int_{-a}^{a} \int_{\gamma_{t_{0}}} e^{i\left[Z(x, t)-Z\left(x^{\prime}, t^{\prime}\right)\right] \xi-\epsilon \xi^{2}} \mathrm{M}^{\prime l}\left[g\left(x^{\prime}\right) \mathrm{L} u\left(x^{\prime}, t^{\prime}\right)\right] d t^{\prime} d x^{\prime} d \xi\right| \\
& =\frac{1}{2 \pi}\left|\int_{0}^{\infty} \int_{-a}^{a} \int_{\gamma_{t}}(1+\xi)^{-2} e^{i\left[Z(x, t)-Z\left(x^{\prime}, t^{\prime}\right)\right] \xi-\epsilon \xi^{2}}\left[1-i \mathrm{M}^{\prime}\right]^{2} \mathrm{M}^{\prime l}\left[g\left(x^{\prime}\right) \mathrm{L} u\left(x^{\prime}, t^{\prime}\right)\right] d t^{\prime} d x^{\prime} d \xi\right|+ \\
& +\frac{1}{2 \pi}\left|\int_{0}^{\infty} \int_{-a}^{a} \int_{\left[l(t), t_{0}\right]}(1+\xi)^{-2} e^{i\left[Z(x, t)-Z\left(x^{\prime}, t^{\prime}\right)\right] \xi-\epsilon \xi^{2}}\left[1-i \mathrm{M}^{\prime}\right]^{2} \mathrm{M}^{\prime l}\left[g\left(x^{\prime}\right) \mathrm{L} u\left(x^{\prime}, t^{\prime}\right)\right] d t^{\prime} d x^{\prime} d \xi\right| \\
& +\frac{1}{2 \pi}\left|\int_{0}^{\infty} \int_{-a}^{a} \int_{\gamma_{t_{0}}}(1+\xi)^{-2} e^{i\left[Z(x, t)-Z\left(x^{\prime}, t^{\prime}\right)\right] \xi-\epsilon \xi^{2}}\left[1-i \mathrm{M}^{\prime}\right]^{2} \mathrm{M}^{\prime l}\left[g\left(x^{\prime}\right) \mathrm{L} u\left(x^{\prime}, t^{\prime}\right)\right] d t^{\prime} d x^{\prime} d \xi\right|
\end{aligned}
$$




$$
\begin{aligned}
& \leq \frac{1}{2 \pi} \int_{0}^{\infty} \int_{-a}^{a} \int_{\gamma_{t}}(1+\xi)^{-2} e^{\left[\Phi\left(t^{\prime}\right)-\Phi(t)\right] \xi-\epsilon \xi^{2}}\left|\left[1-i \mathrm{M}^{\prime}\right]^{2} \mathrm{M}^{\prime l}\left[g\left(x^{\prime}\right) \mathrm{L} u\left(x^{\prime}, t^{\prime}\right)\right]\right| d t^{\prime} d x^{\prime} d \xi+ \\
& +\frac{1}{2 \pi} \int_{0}^{\infty} \int_{-a}^{a} \int_{\left[l(t), t_{0}\right]}(1+\xi)^{-2} e^{\left[\Phi\left(t^{\prime}\right)-\Phi(t)\right] \xi-\epsilon \xi^{2}}\left|\left[1-i \mathrm{M}^{\prime}\right]^{2} \mathrm{M}^{\prime l}\left[g\left(x^{\prime}\right) \mathrm{L} u\left(x^{\prime}, t^{\prime}\right)\right]\right| d t^{\prime} d x^{\prime} d \xi \\
& +\frac{1}{2 \pi} \int_{0}^{\infty} \int_{-a}^{a} \int_{\gamma_{t_{0}}}(1+\xi)^{-2} e^{[\Phi(t)-\Phi(t)] \xi-\epsilon \xi^{2}}\left|\left[1-i \mathrm{M}^{\prime}\right]^{2} \mathrm{M}^{\prime l}\left[g\left(x^{\prime}\right) \mathrm{L} u\left(x^{\prime}, t^{\prime}\right)\right]\right| d t^{\prime} d x^{\prime} d \xi .
\end{aligned}
$$

Observando que $\Phi\left(t^{\prime}\right)-\Phi(t)<0$ para $t^{\prime} \in \gamma_{t},\left[l(t), t_{0}\right]$ e $\gamma_{t_{o}}$ respectivamente, procedendo como no caso anterior obtemos

$$
\begin{aligned}
\left|\mathrm{M}^{l} \mathcal{K}_{\epsilon}^{+}(x, t)\right| & \leq \text { const } \cdot \sup _{\bar{U}}\left|[1-i \mathrm{M}]^{2} \mathrm{M}^{l}[g(x) \mathrm{L} u(x, t)]\right| \int_{0}^{\infty}(1+\xi)^{-2} d \xi \\
& \leq \text { const } \cdot h^{2} 8^{s}\left(4^{s} h\right)^{l}(l !)^{s} .
\end{aligned}
$$

Por outro lado

$$
\begin{aligned}
& \mathrm{M}^{l} \mathcal{H}_{\epsilon}^{+}(x, t)=\partial_{x}^{l} \mathcal{H}_{\epsilon}^{+}(x, t) \\
& =\partial_{x}^{l}\left[\frac{1}{2 \pi} \int_{0}^{\infty} \int_{-a}^{a} e^{i\left(x-x^{\prime}\right) \xi-\left(\Phi(t)-\Phi\left(l\left(t_{0}\right)\right)\right) \xi-\epsilon \xi^{2}} g\left(x^{\prime}\right) u\left(x^{\prime}, l\left(t_{0}\right)\right) d x^{\prime} d \xi\right] \\
& =\frac{1}{2 \pi} \int_{0}^{\infty} \int_{-a}^{a} \partial_{x}^{l}\left[e^{i\left(x-x^{\prime}\right) \xi-\left(\Phi(t)-\Phi\left(l\left(t_{0}\right)\right)\right) \xi-\epsilon \xi^{2}}\right] g\left(x^{\prime}\right) u\left(x^{\prime}, l\left(t_{0}\right)\right) d x^{\prime} d \xi .
\end{aligned}
$$

Como $l(t) \in \Sigma$ segue que $\Phi(t)>0>\Phi\left(t_{0}\right)$ e $\Phi(l(t))=0$. Assim

$$
\begin{aligned}
\Phi(t)-\Phi\left(l\left(t_{0}\right)\right) & \geq \Phi\left(t_{0}\right)-\Phi\left(l\left(t_{0}\right)\right) \\
& \geq C \delta\left(l\left(t_{0}\right)\right)^{\sigma} \\
& \geq C\left|t_{0}-l\left(t_{0}\right)\right|^{\sigma}
\end{aligned}
$$

Por outro lado, como $\Phi\left(l\left(t_{0}\right)\right) \leq 0$ segue que

$$
\begin{aligned}
\Phi(t)-\Phi\left(l\left(t_{0}\right)\right) & \geq \Phi(t) \\
& =\Phi(t)-\Phi(l(t)) \\
& \geq C \delta(t)^{\sigma} \\
& \geq C|t-l(t)|^{\sigma} .
\end{aligned}
$$


Portanto

$$
\Phi(t)-\Phi\left(l\left(t_{0}\right)\right) \geq C \max \left\{\left|t_{0}-l\left(t_{0}\right)\right|,|t-l(t)|\right\}^{\sigma} .
$$

Vamos verificar que

$$
\max \left\{\left|t_{0}-l\left(t_{0}\right)\right|,|t-l(t)|\right\} \geq d^{\prime}
$$

Se $|t-l(t)|<d^{\prime}$ então $\operatorname{dist}(l(t), \partial \Theta)>d^{\prime}$ pois $\operatorname{dist}(l(t), \partial \Theta) \geq 2 d^{\prime}$. Portanto, pela nossa escolha de $t_{0}$, temos $\operatorname{dist}\left(t_{0}, \partial \Theta\right)>d^{\prime}$ e $l\left(t_{0}\right) \in \partial \Theta$ donde $\left|t_{0}-l\left(t_{0}\right)\right|>d^{\prime}$.

Logo, usando (2.14) e (2.13),

$$
\begin{aligned}
\left|\mathrm{M}^{l} \mathcal{H}_{\epsilon}^{+}(x, t)\right| & \leq \frac{1}{2 \pi}\left|\int_{0}^{\infty} \int_{-a}^{a}(i)^{l} \xi^{l} e^{i\left(x-x^{\prime}\right) \xi-d \xi-\epsilon \xi^{2}} g\left(x^{\prime}\right) u\left(x^{\prime}, l\left(t_{0}\right)\right) d x^{\prime} d \xi\right| \\
& \leq \frac{1}{2 \pi} \int_{0}^{\infty} \int_{-a}^{a} \xi^{l} e^{-\left(d \xi+\epsilon \xi^{2}\right)}\left|g\left(x^{\prime}\right) u\left(x^{\prime}, l\left(t_{0}\right)\right)\right| d x^{\prime} d \xi .
\end{aligned}
$$

Procedendo como no caso anterior, obtemos que

$$
\left|\partial_{x}^{l} \mathcal{H}_{\epsilon}^{+}(x, t)\right| \leq \text { Const } \cdot\left(\frac{2}{d}\right)^{l} l !
$$

$\underline{2^{\circ}}$ caso) $t \in \Sigma$ ( portanto $\Phi(t)=0$ ). Neste caso escolhemos $t_{0}$ arbitrariamente próximo de $t$ satisfazendo as condições:

- $\Phi\left(t_{0}\right)<0$ (lembre que $\Phi$ não tem mínimo local).

- $t_{0} \in V$, onde $V$ é uma vizinhança aberta convexa de $t$ na qual $\Phi(s)<0$, $\forall s \in V$.

- $\operatorname{Se} \operatorname{dist}(t, \partial \Theta)>d^{\prime}$ então supomos que $\operatorname{dist}\left(t_{0}, \partial \Theta\right)>d^{\prime}$.

Note que, com a primeira condição acima, $l\left(t_{0}\right) \in \partial \Theta$. Aqui escolhemos $\gamma=\left[t, t_{0}\right] \cup \gamma_{t_{0}}$, onde $\gamma_{t_{0}}$ é como no lema 8 e $\left[t, t_{0}\right]$ é o segmento que une $t$ 
$\operatorname{com} t_{0}$. Então

$$
\begin{aligned}
\mathrm{M}^{l} \mathcal{K}_{\epsilon}^{+}(x, t) & =\frac{1}{2 \pi} \int_{0}^{\infty} \int_{-a}^{a} \int_{\gamma} e^{i\left[Z(x, t)-Z\left(x^{\prime}, t^{\prime}\right)\right] \xi-\epsilon \xi^{2}} \mathrm{M}^{\prime l}\left[g\left(x^{\prime}\right) \mathrm{L} u\left(x^{\prime}, t^{\prime}\right)\right] d x^{\prime} d t^{\prime} d \xi \\
& =\frac{1}{2 \pi} \int_{0}^{\infty} \int_{-a}^{a} \int_{\left[t, t_{0}\right]} e^{i\left[Z(x, t)-Z\left(x^{\prime}, t^{\prime}\right)\right]-\epsilon \xi^{2}} \mathrm{M}^{\prime l}\left[g\left(x^{\prime}\right) \mathrm{L} u\left(x^{\prime}, t^{\prime}\right)\right] d x^{\prime} d t^{\prime} d \xi \\
& +\frac{1}{2 \pi} \int_{0}^{\infty} \int_{-a}^{a} \int_{\gamma_{t_{0}}} e^{i \xi\left[Z(x, t)-Z\left(x^{\prime}, t^{\prime}\right)\right] \xi-\epsilon \xi^{2}} \mathrm{M}^{\prime l}\left[g\left(x^{\prime}\right) \mathrm{L} u\left(x^{\prime}, t^{\prime}\right)\right] d x^{\prime} d t^{\prime} d \xi
\end{aligned}
$$

Portanto, procedendo como acima obtemos as estimativas para $\left|\mathrm{M}^{l} \mathcal{K}_{\epsilon}^{+}(x, t)\right|$ e $\mathrm{M}^{l} \mathcal{H}_{\epsilon}^{+}(x, t)$.

Raciocínio análogo pode ser aplicado utilizando as curvas dadas pelas soluções de

$$
\left\{\begin{array}{cc}
\dot{\gamma}_{t}= & +\frac{\nabla \Phi}{|\nabla \Phi|}\left(\gamma_{t}(\tau)\right) \\
\gamma_{t}(0)= & t
\end{array}\right.
$$

e usando a hipótese que $\Phi$ não tem máximo local para obter as estimativas análogas para $\mathrm{M}^{l} \mathcal{K}_{\epsilon}^{-}, \mathrm{M}^{l} \mathcal{H}_{\epsilon}^{-}$.

\section{Demonstração do corolário :}

$\triangleright \quad$ Se $\Phi$ for analítico em $U$ então pela proposição 2.2 se satisfaz a condição (2.8) e levando em conta a observação 5 se conclui o corolário. 


\section{Capítulo 3}

\section{Exemplo de um sistema que é analítico hipoelíptico, não hipoelíptico $\mathcal{C}^{\infty}$ e não $G^{S}$ hipoelíptico para $s>1$}

Na presente seção discutimos a hipoelipticidade $G^{s}$ do seguinte exemplo, que é conhecido não ser hipoelíptico $\mathcal{C}^{\infty}$ (cf. [M], exemplo 16 e Proposição 23) mas ser analítico hipoelíptico (cf. [BT], Teorema 6.1):

$$
\begin{aligned}
& \mathrm{L}_{1}=\frac{\partial}{\partial t_{1}}+i\left[3 \frac{\partial}{\partial x_{1}}-\left(4 t_{1} t_{2}+3\right) t_{1}^{2} \frac{\partial}{\partial x_{2}}\right] \\
& \mathrm{L}_{2}=\frac{\partial}{\partial t_{2}}-i t_{1}^{4} \frac{\partial}{\partial x_{2}}
\end{aligned}
$$

Em (3.1), $m=n=2$. Os correspondentes $Z_{k}$ (cf. (2.5)) são:

$$
\begin{aligned}
& Z_{1}=x_{1}-3 i t_{1}, \\
& Z_{2}=x_{2}+i\left(t_{1} t_{2}+1\right) t_{1}^{3} .
\end{aligned}
$$

Escolhemos $\Omega$ vizinhança aberta da origem em $\mathbf{R}^{4}$ com fronteira $\mathcal{C}^{\infty}$. 
Teorema 30 sistema (3.1) não é $G^{s}$-Hipoelíptico em $\Omega$ para $s>1$.

Lema 9 Se $\mathrm{L}$ é $G^{s}$-Hipoelíptico em $\Omega$ então para todo $W \subset \subset \Omega$ com fronteira suave e $h>0$ existem $\sigma>0$ e $C>0$ tais que

$$
\begin{gathered}
\sup _{(x, t) \in \bar{W},|\alpha|>0} \frac{\left|\partial^{\alpha} u(x, t)\right|}{\sigma^{|\alpha|}|\alpha !|^{s}} \leq C\left\{\sup _{(x, t) \in \bar{\Omega}}|u(x, t)|+\sup _{(x, t) \in \bar{\Omega},|\alpha|>0} \frac{\left|\partial^{\alpha} \mathrm{L} u(x, t)\right|}{h^{|\alpha|}|\alpha !|^{s}}\right\} \\
\forall u \in \mathcal{C}^{\infty}(\bar{\Omega}) .
\end{gathered}
$$

\section{Demonstração :}

$\triangleright \quad$ Dada $u \in \mathcal{C}^{0}(\Omega)$ com $\operatorname{L} u \in \wedge^{1} G^{s}(\Omega)$ temos que $u \in G^{s}(\Omega)$. Assim para todo $W \subset \subset \Omega$ com fronteira suave, $u \in \mathcal{C}^{0}(\bar{\Omega})$ com $L u \in G^{s}(\bar{\Omega})$ implica $u \in G^{s}(\bar{W})$. Dado $h>0$, denotaremos por

$$
F=\left\{u \in \mathcal{C}^{0}(\bar{\Omega}): L u \in \wedge^{1} G^{s, h}(\bar{\Omega})\right\}
$$

munido da topologia do gráfico. Pela hipoelipticidade de $\mathrm{L}$, repetindo o argumento na demonstração da proposição 1.1, existe $\sigma>0$ tal que $F \subset G^{s, \sigma}(\bar{W})$. Por outro lado, sendo $G^{s, h}(\bar{\Omega})$ um espaço de Banach, o teorema do gráfico fechado implica que $i: F \rightarrow G^{s, \sigma}(\bar{W})$ é contínua. Então existe $C>0$ tais que se cumpre a desigualdade (3.3).

\section{Demonstração do teorema :}

$\triangleright$ Tomamos

$$
u(x, t)=g\left(t_{1}\right) e^{i Z(x, t) \cdot \xi}
$$

onde $Z(x, t) \in \mathbb{C}^{2}$ foi definido em (3.2), $\xi \in \mathbf{R}^{2}$ a ser escolhido depois e $g \in G_{c}^{s, h_{1}}(\mathbf{R})$ não negativa, $h_{1}$ também a ser escolhida. Temos

$$
|u(x, t)|=g\left(t_{1}\right) e^{-\Phi(t) \cdot \xi} .
$$

Escolhemos $\xi_{1}=c^{2} \rho, \xi_{2}=\rho, c>0$ pequeno, $\rho>0$ grande. Por (3.2)

$$
\frac{\Phi(t) \cdot \xi}{\rho} \doteq B\left(t_{1}, t_{2}, c\right)
$$


onde usamos a notação

$$
B(s, a, c)=s^{3}+a s^{4}-3 c^{2} s .
$$

Note que

$$
c^{-3} B(c s, a, c)=s^{3}+a c s^{4}-3 s .
$$

Vamos descrever brevemente o gráfico da curva $y_{2}=c^{-3} B\left(c y_{1}, a, c\right)$ numa vizinhança $V \times U \subset \Omega$ da origem. Dentre as raízes temos 0 e

$$
\varrho_{ \pm}= \pm \sqrt{3}+\theta_{ \pm}(a c)
$$

$\operatorname{com} \theta_{ \pm}$analítica numa vizinhança de zero e anulando-se em zero. De fato, $c^{-3} B\left(c y_{1}, a, c\right)=s P(s, a c) \operatorname{com} P(s, a c)=\left[\left(s^{2}-3\right)+a c s^{3}\right]$ então temos $p( \pm \sqrt{3}, 0)=0$ e $\partial P( \pm \sqrt{3}, 0) / \partial s \neq 0$. Assim o resultado segue do teorema da função implícita.

Agora suponha que $\mu$ é a quarta raiz, então

$$
(s-\mu)\left(s-\sqrt{3}-\theta_{+}(a c)\right)\left(s+\sqrt{3}-\theta_{-}(a c)\right)=0 .
$$

Portanto

$$
\mu\left(\sqrt{3}+\theta_{+}(a c)\right)\left(\sqrt{3}-\theta_{-}(a c)\right)=-3 / a c
$$

e assim a quarta raiz é da ordem de $-\frac{1}{a c}$. Duas das raízes da equação

$$
3 y_{1}^{2}-3+4 a c y_{1}^{3}=0
$$

são da forma

$$
\gamma_{ \pm}= \pm 1+\psi_{ \pm}(a c)
$$

$\operatorname{com} \psi_{ \pm}$analítica numa vizinhança de zero e anulando-se em zero. A outra raiz é da ordem de $-\frac{3}{4 a c}$. A função $y_{2}=y_{1}^{3}-3 y_{1}+a c y_{1}^{4}$ tem um mínimo em $\gamma_{+}$, igual a $-\mu$ :

$$
\mu=3 \gamma_{+}-\gamma_{-}^{3}-a c \gamma_{+}^{4} .
$$


Limitamos a variação de $y_{1}$ ao intervalo $\left[\gamma_{-}, 2\right]$, e $c$ pequeno tal que $-\frac{1}{a c}$ e $-\frac{3}{4 a c}$ estão fora deste intervalo e $\gamma_{-}<0<\gamma_{+}<\varrho_{+}<2$.

Agora tomamos $g\left(t_{1}\right)=g_{0}\left(t_{1} / c\right)$, e

$$
\text { supp } \left.g_{0} \subset\right]-3 / 2,+3\left[, \quad g_{0}=1 \mathrm{em} \quad[-c, 2 c]\right. \text {. }
$$

Por (3.8) e tomando $c$ suficientemente pequeno com $|a| \leq R_{0}, R_{0}$ o raio de $U$, temos:

$$
\sup _{s \in \operatorname{supp}} c_{0}^{\prime} c^{-3} B(c s, a, c) \geq d_{0}>0 .
$$

Dado $W \subset \subset \Omega$ escolho $K=\left\{(x, t) \in V \times U ;\left|t_{1}\right| \leq b, t_{2}=0, x_{2}=0\right\} \subset$ $W, \operatorname{com} b>0$ pequeno. Para $c$ pequeno o ponto $(c, 0,0,0) \in K \mathrm{e}$

$$
B\left(t_{1}, 0, c\right) \geq B(c, 0, c)=-2 c^{3}, \quad \forall t_{1} \in \operatorname{supp} g
$$

\section{Então}

$$
\begin{aligned}
\partial_{x_{2}} u(x, t) & =\partial_{x_{2}}\left(g\left(t_{1}\right) e^{i x \cdot \xi-\Phi(t) \cdot \xi}\right) \\
& =i g\left(t_{1}\right) \rho e^{i x \cdot \xi-\Phi(t) \cdot \xi}
\end{aligned}
$$

Assim,

$$
\left|\partial_{x_{2}} u(x, t)\right|=\left|g\left(t_{1}\right)\right| \rho e^{-\Phi(t) \cdot \xi} \quad t_{1} \in \operatorname{supp} g .
$$

Logo

$$
\sup _{(x, t) \in K}\left|\partial_{x_{2}} u(x, t)\right|=\rho \sup _{(x, t) \in K} e^{-\Phi(t) \cdot \xi}=\rho e^{2 c^{3} \rho} .
$$

Podemos agora tomar o conjunto $\overline{V \times U}$ como sendo da forma $\overline{V^{\prime}} \times K_{0}^{i}$, com $K_{0}^{\prime}$ o quadrado $\left|t_{j}\right| \leq R_{0}, j=1,2$ e $\overline{V^{\prime}}$ uma vizinhança compacta da origem. Se $t_{1} \in \operatorname{supp} g$ e $t_{2}=a$, temos

$$
B\left(t_{1}, t_{2}, c\right) \geq B\left(\gamma_{+}, a, c\right)=-c^{3} \mu
$$

e $\mu=2+O(c)$. De isto e de (3.5) temos que

$$
\sup _{\overline{V^{\prime}} \times K_{0}^{\prime}}|u| \leq e^{2 c^{3} \rho+O\left(c^{4}\right) \rho} .
$$


Por outro lado, seja $\Phi^{\#}$ extensão holomorfa de $i Z(x, t) \cdot \xi$ definida numa vizinhança complexa de $\overline{V^{\prime}} \times K_{0}^{\prime}$ e seja $r$ suficientemente pequeno a ponto de tornar os poli-discos $\triangle_{1}(x, r) \times \triangle_{2}(t, r),(x, t) \in \overline{V^{\prime}} \times K_{0}^{\prime}$, contidos nesta vizinhança complexa.

Da fórmula integral de Cauchy segue que para $(x, t) \in \overline{V^{\prime}} \times K_{0}^{\prime}$,

$$
\partial^{\beta} e^{i Z(x, t) \cdot \xi}=\frac{\beta !}{(2 \pi i)^{2}} \int_{\partial \triangle_{1}(x, r) \times \partial \triangle_{2}(t, r)} \frac{e^{\Phi^{\#}(z)}}{(z-(x, t))^{\beta+(1,1,1,1)}} d z
$$

donde

$\left|\partial^{\beta} e^{i Z(x, t) \cdot \xi}\right| \leq \exp \left[\sup \left\{\operatorname{Re} \Phi^{\#}(z) ; z \in \partial \triangle_{1}(x, r) \times \partial \triangle_{2}(t, r)\right\}\right] \cdot r^{-|\beta|} \cdot \beta !$

Dado que

$$
\begin{aligned}
-\sup \{\operatorname{Re}[i Z(x, t) \cdot \xi] & \left.=\rho B\left(t_{1}, t_{2}, c\right): t_{1} \in \operatorname{supp} g_{0}^{\prime}\right\} \\
& =\rho \sup B(c s, a, c) \quad,|a| \leq R_{0}, s \in \operatorname{supp} g_{0}^{\prime} \\
& \geq \rho d_{0} c^{3},
\end{aligned}
$$

podemos, por continuidade, diminuindo $r$ (se necessário), assumir que $\sup \left\{\operatorname{Re} \Phi^{\#}(z): z \in \partial \triangle_{1}(x, r) \times \partial \triangle_{2}(t, r), t_{1} \in \operatorname{supp} g_{0}^{\prime}\right\} \leq-\rho d_{0} c^{3} / 2$

Observe que $L u=g^{\prime}\left(t_{1}\right) e^{i Z(x, t) \cdot \xi}=g^{\prime}\left(t_{1}\right) e^{i x \cdot \xi-\Phi(t) \cdot \xi}$ e portanto

$$
\begin{aligned}
\sup _{\overline{V^{\prime}} \times K_{0}^{\prime}}\left|\partial^{\alpha} L u\right| & \leq \sum_{\beta \leq \alpha}\left(\begin{array}{c}
\alpha \\
\beta
\end{array}\right) \sup _{\operatorname{supp}_{g^{\prime}}}\left|\partial^{\beta} g^{\prime}\left(t_{1}\right)\right| \sup _{\overline{V^{\prime}} \times\left(K_{0}^{\prime} n \operatorname{supp} g^{\prime}\right)}\left|\partial^{\alpha-\beta} e^{i Z(x, t) \cdot \xi}\right| \\
& \leq C \sum_{\beta \leq \alpha}\left(\begin{array}{l}
\alpha \\
\beta
\end{array}\right)(\beta !)^{s} h_{1}^{|\beta|} e^{-\frac{c^{3} d_{0} \rho}{2}}(\alpha-\beta) ! h_{2}^{|\alpha-\beta|} \quad h_{2}=1 / r \\
& \leq C e^{-\frac{c^{3} d_{0} \rho}{2}} \sum_{\beta \leq \alpha} \frac{\alpha !}{\beta !}(\beta !)^{s} h_{1}^{|\beta|} h_{2}^{|\alpha-\beta|} \\
& \leq C e^{-\frac{c^{3} d_{0} \rho}{2}}(\alpha !)^{s} \max \left\{h_{1}, h_{2}\right\}^{|\alpha|} C_{1}^{|\alpha|} .
\end{aligned}
$$


Seja $h_{*}=\max \left\{h_{1}, h_{2}, C_{1}\right\}$, então

$$
\left|\partial^{\alpha} \operatorname{Lu}\right| \leq C e^{-\frac{c^{3} d_{0} \rho}{2}} h_{*}^{|\alpha|}(\alpha !)^{s} .
$$

Portanto, se tomamos $h=h_{*}$, obtemos

$$
\sup _{\overline{V^{\prime}} \times K_{0}^{\prime}} \frac{\left|\partial^{\alpha} L u\right|}{h^{|\alpha|}(\alpha !)^{s}} \leq C e^{-\frac{c^{3} d_{0} \rho}{2}} .
$$

Agora, pelos resultados (3.10), (3.11) e (3.12) vemos que (3.3) implica

$$
\rho e^{2 c^{3} \rho} \leq \sigma C\left\{e^{2 c^{3} \rho+O\left(c^{4}\right) \rho}+e^{-\frac{c^{3} d_{0} \rho}{2}}\right\}
$$

assim

$$
\rho \leq \sigma C\left\{e^{O\left(c^{4}\right) \rho}+e^{-\left(2+d_{0} / 2\right) c^{3} \rho}\right\}
$$

Para $\rho=1 / c^{4}$ e fazendo $c>0$ ir para zero; o lado direito é limitado, mas isto não é certo para o lado esquerdo. 


\section{Simbologia}

- as variáveis

$$
\begin{aligned}
& x=\left(x_{1}, \ldots, x_{m}\right) \in \mathbb{R}^{m}, \xi=\left(\xi_{1}, \ldots, \xi_{m}\right) \in \mathbb{R}_{m}, \\
& \dot{\xi}=\left(\dot{\xi}_{1}, \ldots, \dot{\xi}_{m}\right) \in S^{m-1}, t=\left(t_{1}, \ldots, t_{n}\right) \in \mathbb{R}^{n} \\
& z=\left(z_{1}, \ldots, z_{m}\right) \in \mathbb{C}^{m}
\end{aligned}
$$

- os domínios

$$
B \ni x, \Theta \ni t, P \ni \dot{\xi}, U \ni(x, t), \Gamma(P) \ni \xi
$$

- os espaços de funções

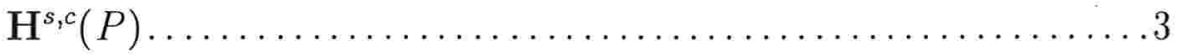

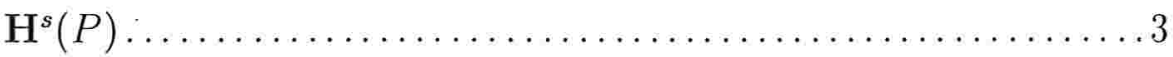

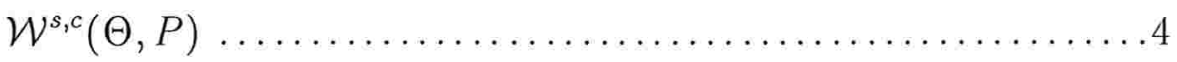

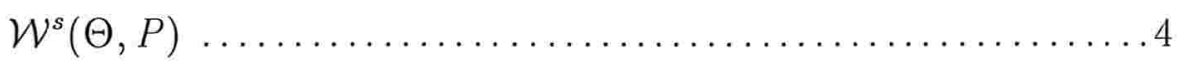

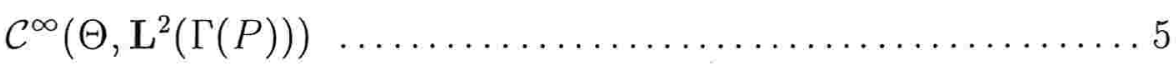

- operador:

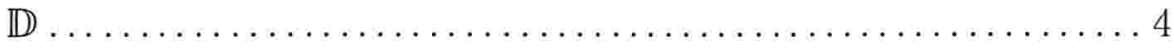

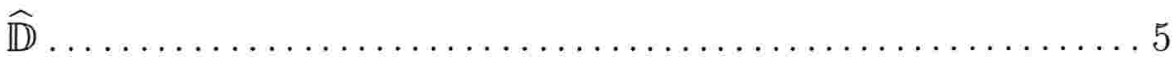

- Gevrey usual

$G^{s, h}(\bar{U}), G^{s}(\bar{U}), G^{s}(V) \ldots \ldots \ldots \ldots \ldots \ldots \ldots \ldots \ldots \ldots \ldots \ldots \ldots \ldots \ldots \ldots, 29,30$

- Gevrey segundo a estrutura localmente integrável $\mathrm{G}_{\mathrm{ML}}^{s, h}(V), \mathrm{G}_{\mathrm{ML}}^{s}(V)$. 


\section{Referências Bibliográficas}

[A] C. Asano, Hipoelipticidade de uma classe de sistemas sobredeterminados. UnIVERSIDAde DE SÃo PAUlo, Tese de Mestrado-1991

[BT] M. S. Baouendi e F. Treves, A property of the functions and distributions annihilated by a locally integrable system of complex vector fields. AnNAls of MATh., 113 (1981), 387-421

[BT1] M. S. Baouendi e F. Treves, A Microlocal version of Bochner's tube theorem. Indiana University Mathematics Journal, vol. 31, No. 6 (1982), 885-895

[C] P. A. Caetano, Classes de Gevrey em Estruturas Hipo-analíticas. Universidade de São Paulo, Tese de Doutorado-2000

[Ch] M. Christ, Intermediate optimal Gevrey exponents occur. Сомм. In Partial Diff. EQ., 22(3\&4)(1997), 359-379

[G] A. Grothendieck, Espaces vectoriels topologiques. SocIEDADE DE Matemática de S. Paulo (3.A Edição), São Paulo-1964

[K] H. Komatsu, Ultradistributions I. Structures theorems and a caracterization. J. FAC.SCI.UNIV.TOKIO, SEC IA, 19(1973), 321-352 
[Ko] H. Komatsu, Projective and injective limits of weakly compact sequences of locally convex spaces. J. MATH. Soc. JAPAN, 19(3)(1967), 366-383

[L] S. Lojasiewicz, Ensembles Semi-Analytiques. Institut des Hautes Etudes Scientifiques. Bures-sur-Yvette (Seine-et-Oise), France. (notas mimeografadas)

[M] H. M. Maire, Hypoelliptic overdetermined systems of partial differential equations. Comm. IN PARTial DifF. EQ., 5(4)(1980), 331-380

[T] F. Treves, Study of a model in the theory of complexes of pseudodifferential operators. AnNals of Math., 104 (1976), 325-356

[T1] F. Treves, Hypoelliptic partial differential equations of principal type. Comm. Pure Applied Math., 24 (1971), 631-670

[T2] F. Treves, Hypo-analytic structures (local theory). PRINCETON University Press, Princeton, NJ, 1992 